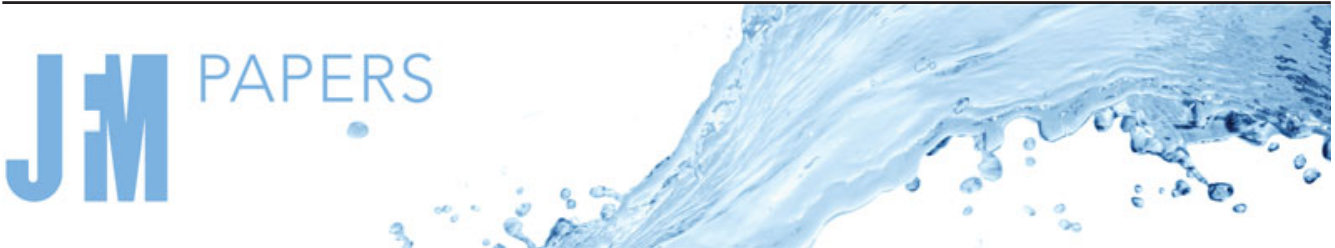

\section{Aerofoil wake-induced transition characteristics on a flat-plate boundary layer}

\author{
Dhamotharan Veerasamy ${ }^{1,}$, , Chris J. Atkin ${ }^{1,2}$ and \\ Sathiskumar A. Ponnusami ${ }^{1}$ \\ ${ }^{1}$ Department of Mechanical Engineering and Aeronautics, City, University of London, \\ London EC1V 0HB, UK \\ ${ }^{2}$ School of Engineering, University of East Anglia, Norwich NR4 7TJ, UK
}

(Received 7 May 2020; revised 3 March 2021; accepted 19 May 2021)

This paper presents an experimental investigation of the characteristics of laminarturbulent transition occurring on a flat-plate boundary layer due to the interaction with a non-impinging aerofoil wake. Previous studies have tended to focus on transition induced by free-stream turbulence or by the wake of a circular cylinder, both of which exhibit different forcing characteristics to the present experimental arrangement. A tripped NACA 0014 aerofoil was used to generate a fully turbulent wake, upstream of and at various heights above a laminar, flat-plate boundary layer, in the UK National Low-turbulence Wind Tunnel at City, University of London. Hot-wire measurements conducted in the pre-transitional region reveal the wall-normal and spanwise structure of the disturbances induced within the boundary layer and the rate of growth of disturbance energy. Disturbance profiles generally (but not uniquely) follow the non-modal distribution obtained from transient growth theory, but energy growth rates are mainly exponential rather than algebraic. Energy spectra demonstrate the existence of mixed transitional features (both natural and bypass) in the boundary layer. Two-point spatial correlations reveal the presence of a streaky structure, but with spanwise scale much larger than the boundary layer thickness, in contrast to the trends seen in free-stream turbulence-induced bypass transition and cylinder wake-induced transition. The gap between aerofoil and flat plate affects both the evolution of non-modal disturbance profile and the appearance of the streaky structure; the spacing of the streaks was also found to scale with the vertical gap between aerofoil and flat plate. Overall, the combination of observed characteristics is quite different from the forced transition mechanisms previously reported in the literature.

Key words: boundary layer receptivity, wakes, transition to turbulence

$†$ Email address for correspondence: dhamotharan.veerasamy.1@ city.ac.uk

(C) The Author(s), 2021. Published by Cambridge University Press. This is an Open Access article, distributed under the terms of the Creative Commons Attribution licence (http://creativecommons.org/ licenses/by/4.0/), which permits unrestricted re-use, distribution, and reproduction in any medium, provided the original work is properly cited. 


\section{Introduction}

Wake-boundary layer interactions are common in multi-element aerofoil and turbomachinery flows. Laminar-turbulent transition caused by the interaction of the upstream aerofoil/blade wake with the downstream aerofoil/blade boundary layer is referred to as wake-induced transition. In the past few decades, there has been much research carried out on this topic to elucidate the underlying transition mechanisms. In most of the fundamental studies, the upstream wake was generated by a circular cylinder rather than an aerofoil/blade (Kyriakides et al. 1999; Ovchinnikov, Piomelli \& Choudhari 2006; Pan et al. 2008; Coull \& Hodson 2011; Mandal \& Dey 2011; He, Wang \& Pan 2013). This choice of forcing source may have been inspired by the study of Pfeil \& Herbst (1979), who reported that, in the case of turbomachinery studies, the global wake characteristics (mean velocity profile and drag) of blade and circular cylinder cascades were nearly the same, thus making it reasonable to choose the circular cylinder for the generation of the wake. Following this study, turbomachinery researchers investigated unsteady wake-induced transition by simulating the periodically passing upstream wakes using a moving circular cylinder arrangement. A detailed review of transition due to unsteady wake interactions can be found in Hodson \& Howell (2005) and Durbin \& Wu (2007).

On the other hand, for multi-element aerofoils (high-lift devices), fundamental transition studies have mainly considered a steady/static wake interacting with the downstream boundary layer. Squire (1989), reports that, up to that time, steady wake-boundary layer interaction studies were conducted with a variety of upstream wake generating bodies (circular cylinder, aerofoil and flat plate), but the investigations were more focused on merging boundary layers and how this affected the turbulent statistics. Research into wake-induced transition has been a more recent trend, led by the turbomachinery community as referenced above and primarily employing circular cylinders as the upstream wake generating body. The ascertained laminar-turbulent transitional characteristics in the static cylinder wake-boundary layer interaction are discussed below.

Kyriakides et al. (1999) investigated cylinder wake-induced transition in a downstream flat-plate boundary layer. The coherent structures in the wake were found to behave like spanwise vortices, interacting with the flat-plate boundary layer from a distance and inducing a secondary spanwise vortex in the near-wall flow. However, direct numerical simulation on a similar set-up (Ovchinnikov et al. 2006) did not produce the secondary spanwise vortex in the boundary layer, instead exhibited the presence of streak-like structures aligned in the streamwise direction, a feature observed in free-stream turbulence (FST) induced bypass transition (Matsubara \& Alfredsson 2001). These discrepancies prompted Pan et al. (2008) to use flow visualisation to confirm the presence of a secondary, spanwise vortex in the initial stage of transition, followed by destabilisation into hairpin shaped structures, ultimately resulting in streak formation and transition onset.

Mandal \& Dey (2011) further confirmed the observations of Pan et al. (2008) through detailed particle image velocimetry measurements and reported three distinct flow characteristics: a self-similar wall-normal profile of $u_{r m s}\left(u_{r m s}\right.$ stands for root mean square of the fluctuating velocity); a streak spacing of the order of the boundary layer thickness; and a linear increase in streamwise disturbance energy with downstream distance. These are all features of FST-induced bypass transition (Matsubara \& Alfredsson 2001; Fransson, Matsubara \& Alfredsson 2005). On the whole, the literature suggests that the initial stage of the cylinder wake-induced transition process is dominated by the secondary spanwise vortices and the later stage possesses the features of FST-induced bypass transition. It is 
clear that the formation of the secondary spanwise vortices in the initial stages of transition can be ascribed to vortex shedding from the cylinder wake, an inherent characteristic of a bluff body.

In practical applications, the upstream wakes tend to originate from a slender body (aerofoil/blade), and the wake disturbance characteristics are entirely different from the cylinder wake, particularly in multi-element high-lift systems and turbofan engines where chord Reynolds numbers are of the order of $10^{6}$ (Hourmouziadis 1989; Spaid 2000). At such high Reynolds numbers, the wake of the aerofoil/blade is expected to be fully turbulent with no large scale unsteadiness (i.e. no vortex shedding). It is anticipated that this difference in wake characteristics would lead to different transition mechanisms. The goal of the present research is therefore to study wake-induced transition under a more realistic forcing conditions. While retaining the flat-plate arrangement to facilitate measurement of the initial disturbance growth and the associated transition process. To the best of our knowledge, such an experimental set-up involving a fully turbulent aerofoil wake interacting with a flat-plate boundary layer has not been used before to study the fundamental transition process. A very recent study by Delattre et al. (2018) involved an aerofoil-aerofoil combination, but the transitional characteristics are not discussed in great detail. The results obtained from the present experiments were compared with the literature on cylinder wake-induced transition and also FST-induced bypass transition to highlight the essential differences between the various transition mechanisms.

\section{Experimental details}

The present experiment was conducted in the low-turbulence wind tunnel at City, University of London. This is a closed-circuit wind tunnel having a test section dimension $0.924 \times 0.915 \times 3.66 \mathrm{~m}$ (width $\times$ height $\times$ length). The measured FST intensity $\left(T_{u}\right)$ was $0.007 \%$ at $20 \mathrm{~m} \mathrm{~s}^{-1}$ with an empty test section and increased to $0.015 \%$ with the experimental rig installed (within the frequency band of $5 \mathrm{~Hz}$ to $5 \mathrm{kHz}$ ). Such a very low turbulence level is advantageous for transition studies. A flat plate of length $(l) 2255$ $\mathrm{mm}$, width $915 \mathrm{~mm}$ and thickness $10 \mathrm{~mm}$ was mounted vertically at the mid-plane of the test section. The leading edge of the flat plate was designed as an asymmetric wooden biconvex surface, which helps to minimise the pressure gradient on the working side. This model was previously used in several instability experiments, for example Li \& Gaster (2006). The length of the leading edge was $63 \mathrm{~mm}$ and the profile is detailed in Veerasamy (2019), and the remaining section of the flat plate was made of aluminium. The wake was generated using a NACA 0014 aerofoil with chord length $(c)$ of $253 \mathrm{~mm}$, spanning the entire width of the test section and at zero angle of attack . To avoid vortex shedding and to simulate a fully turbulent wake, the aerofoil boundary layer was tripped at $25 \%$ of the chord from the leading edge. This was done using a sandpaper roughness strip with an average roughness height of $425 \mu \mathrm{m}$. The aerofoil was positioned upstream and 'above' the flat plate, as illustrated in figure 1 . To maintain the stagnation point on the measurement side of the leading edge and to maintain the zero pressure gradient on the flat plate, a trailing edge flap of length $110 \mathrm{~mm}$ was installed.

All velocity measurements were carried out using a boundary layer probe with a constant temperature anemometry unit, Dantec DISA 55M01. The sensor used in the probe was a fine tungsten wire of diameter $5 \mu \mathrm{m}$ and length $1.25 \mathrm{~mm}$. Raw hot-wire voltage data were acquired at a sampling rate of $10 \mathrm{kHz}$ for a duration of $30 \mathrm{~s}$ and, using an analogue filter (Krohn-Hite model 3360 series), fluctuation signals alone were extracted within the band of $5 \mathrm{~Hz}-5 \mathrm{kHz}$. To align the probe close to the surface of the flat plate, a laser 


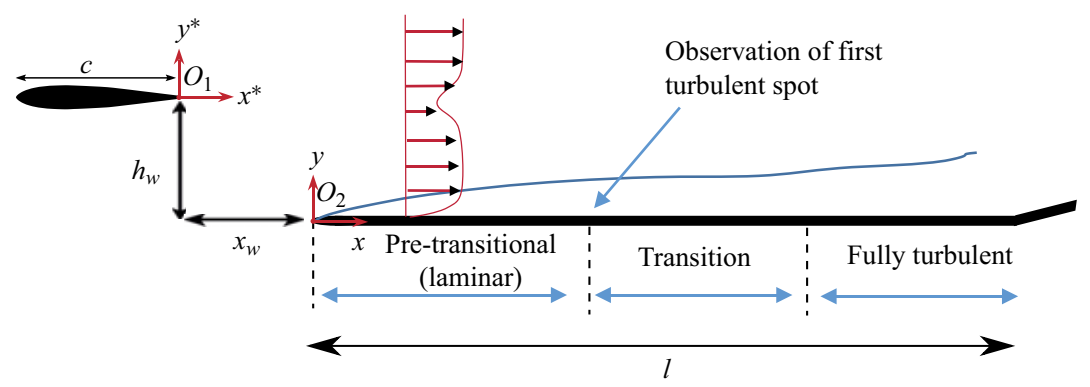

Figure 1. Schematic representation of the experimental set-up.

positioning system was used. The streamwise, wall-normal and spanwise stations were defined using a coordinate system with $x, y$ and $z$ axes respectively, with the origin $\left(\mathrm{O}_{2}\right)$ located at the mid-point of the leading edge of the flat plate. Moreover, for the aerofoil wake measurement (without the flat plate), the coordinate system is denoted as $x^{*}, y^{*}$, and $z^{*}$, with origin $\left(O_{1}\right)$ at the trailing edge of the aerofoil.

The streamwise and lateral gaps between the aerofoil and flat plate are denoted as 'overlap' $\left(x_{w}\right)$ and 'height' $\left(h_{w}\right)$ respectively. This paper presents results for four aerofoil heights $h_{w}=20,40,60$ and $80 \mathrm{~mm}$ but with a constant overlap, $x_{w}$, of $25 \%$ of the aerofoil chord. All experiments were carried out at a free-stream velocity $\left(U_{0}\right)$ of $20 \mathrm{~m} \mathrm{~s}^{-1}$ and corresponding Reynolds number, based on the aerofoil chord $\left(R e_{c}\right)$, of $3.4 \times 10^{5}$. A zero pressure gradient was successfully maintained over the flat plate for all aerofoil heights, as shown in figure 4 of Veerasamy \& Atkin (2020), which paper also describes the raw signal post-processing in greater detail. Due to mechanical constraints, measurements on the flat plate were limited to the region within $900 \mathrm{~mm}$ of the leading edge. Streamwise stations and streamwise velocity are normalised with the flat-plate length $(l=2255)$ and reference free-stream velocity $\left(20 \mathrm{~m} \mathrm{~s}^{-1}\right)$ respectively.

\subsection{Wake characteristics of the aerofoil and measurement regime}

The time-averaged streamwise mean velocity $(\bar{U})$ profile of the wake, the turbulent intensity profile and the spectral distributions obtained at $x^{*} / c=0.2$ in the absence of the flat plate are plotted in figure 2; here, $u_{0}$ is defined as the maximum defect velocity given by $\left(U_{0}-\bar{U}\right)_{\max }$, and $y_{0.5}$ is the lateral position where the defect velocity is $0.5 u_{0}$. Figure $2(a)$ shows that the wake mean velocity follows the plane wake self-similar solution obtained by Wygnanski et al. (1986) except for the near wake profiles, $x^{*} / c=0.04$ and 0.2. During the main experiment the flat plate was positioned at a streamwise offset of $x_{w}=x^{*}=0.25 c$. The approximate relative position of the leading edge of the flat plate and the turbulence level at the leading edge are shown in figure $2(b, c)$. These data are approximate because the measurement of the aerofoil wake at $x^{*} / c=0.2$ was slightly upstream of the flat-plate leading edge, $x_{w}=x^{*}=0.25 c$ in the main experiment. The maximum turbulence intensity at the wake core can observed to be $\approx 12 \%$. For $h_{w}=20 \mathrm{~mm}$ the wake meets the leading edge at $\eta=y^{*} / y_{0.5}^{*}=-3.3$ and it can be assumed that turbulence in the intermittent extremities of the wake will impact directly upon the leading edge. For $h_{w}=40 \mathrm{~mm}$ the leading edge lies at a wake coordinate of $\eta=-6.5$ and it can be assumed that the wake turbulence does not impinge directly upon the leading edge. For these two cases, the effective $T_{u}$ at the leading edge is inferred to be $\approx 0.23 \%$ and $0.04 \%$ respectively. For $h_{w}=60$ and $80 \mathrm{~mm}$ we envisage that the turbulence level would have further decreased 

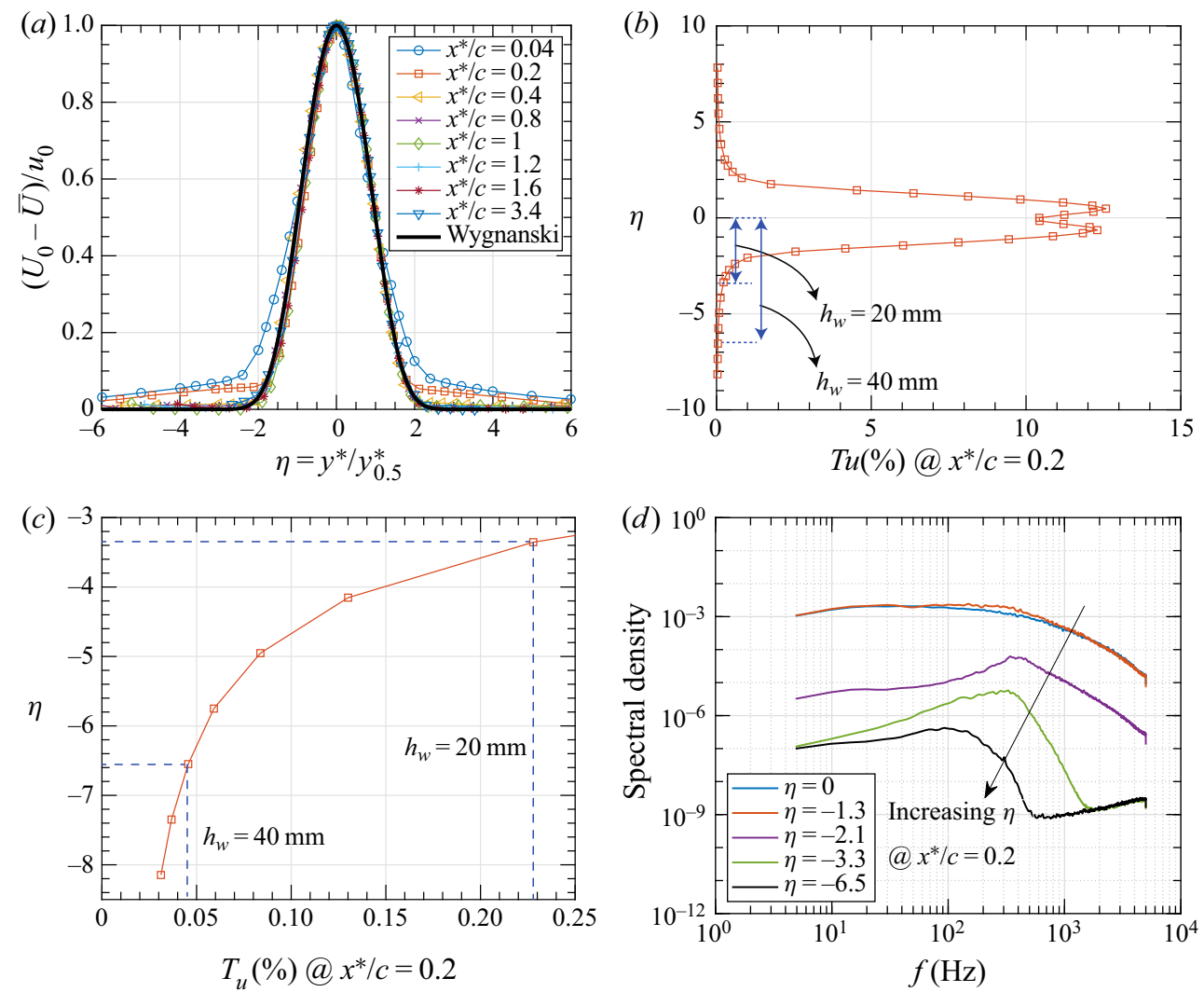

Figure 2. (a) Self-similar mean velocity profiles in the aerofoil wake, measured at various streamwise stations downstream of the trailing edge. The black solid line corresponds to Wygnanski, Champagne \& Marasli (1986). For reference, measured streamwise stations in terms of $x / l$ is $-0.02,-0.005,0.017,0.061,0.083,0.11,0.15$ and 0.35 , respectively, are given in the legend. (b) Turbulent intensity of the wake measured at $x^{*} / c=0.2$, and the $T_{u}$ encountered by the flat-plate leading edge for the height, $h_{w}=20$ and $40 \mathrm{~mm}$ are magnified in $(c)$. (d) Power spectral density of the aerofoil wake at $x^{*} / c=0.2$. These measurement were obtained at $R e_{c}=3.4 \times 10^{5}$, and in the absence of the flat plate.

and encountered the flat-plate leading edge (approximately) closer to the tunnel turbulence intensity, $0.015 \%$. Further, the spectral distribution of wake turbulence at $x^{*} / c=0.2$, shown in figure $2(d)$, does not exhibit any sharp peak, which is an indication of absence of vortex shedding in the present wake. On the other hand, a dominant band of mid-range frequencies is evident some distance away from the edge of the wake $(\eta<-2)$. These band of mid-range frequencies may be associated with the intermittent nature of the turbulent flow at the wake edge, as noted in Veerasamy (2019). Moreover, the disturbance growth and the integral length scale (discussed in §3.1) suggest that these disturbances are anisotropic. Thus, the wake disturbance considered in the present experiments is anisotropic in nature with no large-scale unsteadiness.

The bandwidth of the dominant mid-range frequencies in the spectrum varies with $\eta$. Therefore, the frequency of the disturbance interacting with the downstream boundary layer will vary with the aerofoil height. For instance, figure $2(d)$ shows the variations in dominant disturbance frequency penetrating through the leading edge of the flat plate, where we can infer that, at $\eta=-3.3\left(h_{w}=20\right)$, the dominant frequency, $f=350 \mathrm{~Hz}$ 


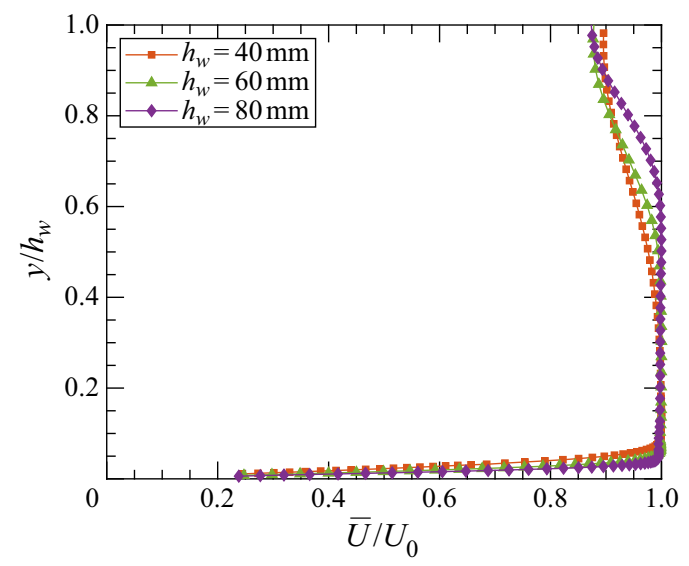

Figure 3. Mean velocity profiles measured at $x / l=0.22$ at different $h_{w}$, showing the potential core $\left(y / h_{w} \approx 0.1-0.4\right)$ between the wake and the boundary layer.
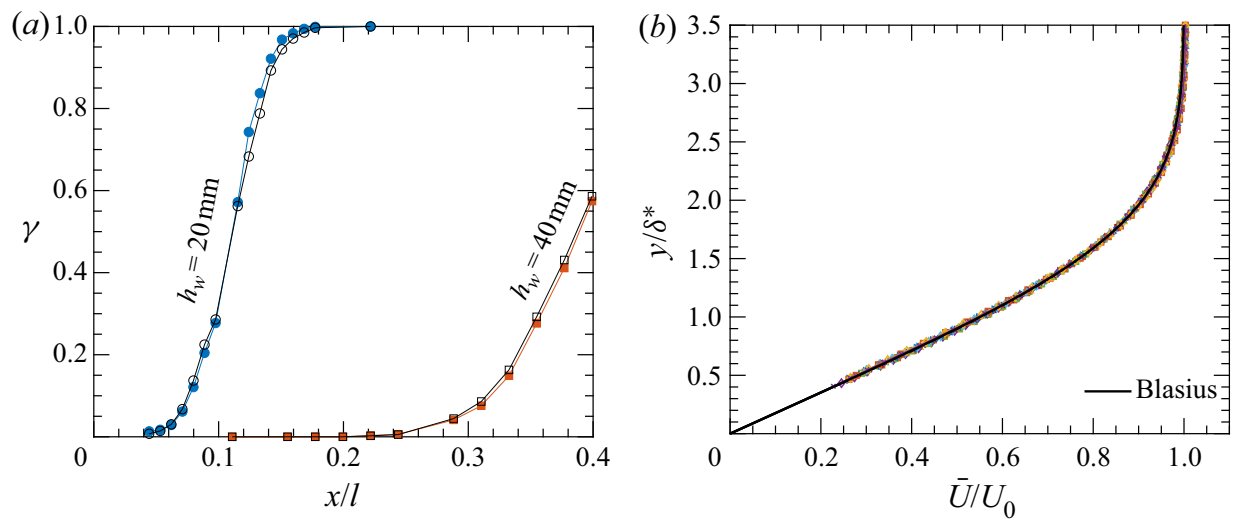

Figure 4. (a) Intermittency distribution on the flat plate obtained at $y / \delta^{*}=0.5$ for $h_{w}=20$ and $40 \mathrm{~mm}$. Curves with solid markers are obtained using a simple rational method proposed in Veerasamy \& Atkin (2020), and those with hollow markers using the dual-slope method of Kuan \& Wang (1990). For $h_{w}=60$ and $80 \mathrm{~mm}$, the flow remains laminar throughout. $(b)$ Mean velocity profiles at the stations given in table 1 compared with the Blasius solution.

is slightly higher than $\eta=-6.5\left(h_{w}=40 \mathrm{~mm}\right), f=150 \mathrm{~Hz}$. For the minimum aerofoil height $\left(h_{w}=20 \mathrm{~mm}\right)$ considered in the present experiment, contact between the wake profile and the leading edge of the flat plate occurs at $\eta=-3.3$. Since $\eta=-3.3$ is slightly beyond the edge of a fully developed wake (figure $2 a$ ), the resulting oncoming velocity at the leading edge is effectively equal to the free-stream velocity $\left(U_{\infty}\right)$. A 'ore' of potential flow develops with increasing aerofoil height $\left(h_{w}=40,60\right.$ and $\left.80 \mathrm{~mm}\right)$, as seen in figure 3, which presents mean velocity profiles obtained at the middle of the measurement domain $(x / l=0.22)$ for three different $h_{w}$. This also shows that disturbances from the wake interact with the flat-plate boundary layer without disturbing its mean velocity profile.

To identify the laminar-turbulent transition on the flat-plate boundary layer, the intermittency $(\gamma)$ distribution was obtained using both the rational approach proposed in Veerasamy \& Atkin (2020) and the dual-slope method (Kuan \& Wang 1990), shown in figure $4(a)$. For $h_{w}=20 \mathrm{~mm}$ transition onset is early at $x / l=0.06$ while for $h_{w}=$ $40 \mathrm{~mm}$ transition onset is at $x / l=0.24$. Here, transition onset is defined as the point 
Transition induced by the upstream aerofoil wake

\begin{tabular}{lccccccc}
$\begin{array}{l}h_{w} \\
(\mathrm{~mm})\end{array}$ & $\begin{array}{c}x \\
(\mathrm{~mm})\end{array}$ & $x / l$ & $\begin{array}{c}\delta \\
(\mathrm{mm})\end{array}$ & $\begin{array}{c}\delta^{*} \\
(\mathrm{~mm})\end{array}$ & $\begin{array}{c}\theta \\
(\mathrm{mm})\end{array}$ & $\mathrm{H}$ & $R e_{\delta^{*}}$ \\
\hline 40 & 250 & 0.11 & 2.50 & 0.75 & 0.29 & 2.59 & 1015 \\
40 & 300 & 0.13 & 2.62 & 0.80 & 0.31 & 2.58 & 1083 \\
40 & 400 & 0.18 & 2.99 & 0.93 & 0.36 & 2.58 & 1259 \\
40 & 500 & 0.22 & 3.19 & 1.00 & 0.39 & 2.56 & 1353 \\
60 & 300 & 0.13 & 2.50 & 0.78 & 0.30 & 2.60 & 920 \\
60 & 500 & 0.22 & 3.16 & 1.02 & 0.39 & 2.61 & 1380 \\
60 & 700 & 0.31 & 3.80 & 1.17 & 0.45 & 2.60 & 1583 \\
60 & 900 & 0.40 & 4.29 & 1.35 & 0.52 & 2.59 & 1827 \\
80 & 300 & 0.13 & 2.57 & 0.83 & 0.32 & 2.61 & 1099 \\
80 & 500 & 0.22 & 3.34 & 1.06 & 0.41 & 2.58 & 1404 \\
80 & 700 & 0.31 & 3.90 & 1.24 & 0.48 & 2.59 & 1642 \\
80 & 900 & 0.4 & 4.30 & 1.40 & 0.54 & 2.59 & 1854
\end{tabular}

Table 1. Boundary layer parameters for different $h_{w} ; x_{w}$ and $U_{0}$ maintained at $0.25 c(63.25 \mathrm{~mm})$ and $20 \mathrm{~m} \mathrm{~s}^{-1}$ throughout. Here, $X / l=0$ is at the flat-plate leading edge. $\delta$ is the wall-normal distance where $\bar{U}=0.99 U_{0}$.

where the intermittency deviates from the value of zero, and the region upstream of the transition onset is termed the 'pre-transitional' region. For $h_{w}=60$ and $80 \mathrm{~mm}$, the flow remains fully laminar $(\gamma=0)$ throughout the measurement domain (up to $x / l=0.4$ ) and consequently the whole domain can be considered as pre-transitional. Boundary layer profiles measured within the pre-transitional region for $h_{w}=40,60$ and $80 \mathrm{~mm}$ follow the Blasius solution, figure $4(b)$. The streamwise measurement stations are given in table 1 along with the derived boundary layer parameters.

\subsection{Correlation measurement technique}

Two-point spatial correlation measurements were conducted using one fixed probe and one traversable probe. The fixed probe was mounted inclined towards the wall which had a longer probe support with a length $150 \mathrm{~mm}$, and the traversable probe support was $70 \mathrm{~mm}$ in length (excluding the aerofoil shaped fairings attached to its rear) mounted parallel to the flat plate with boundary layer sensor probe. Using this two probe set-up, both one-dimensional (1-D) and two dimensional (2-D) spatial correlations were conducted. For 1-D correlation the probes were aligned in the streamwise and wall-normal directions, but offset in the spanwise direction (so $\Delta x, \Delta y=0 ; \Delta z \neq 0$ ). For 2-D correlation the probes were aligned only in the wall-normal direction and were offset in both spanwise and streamwise directions (so $\Delta y=0 ; \Delta x, \Delta z \neq 0$ ). The streamwise separation of the probes introduces the risk of wake interference by one probe on the other. Interference was detected by comparing the spectral characteristics of the single probe measurements with the dual probe measurements. We observed probe interference effects for $\Delta z<6 \mathrm{~mm}$, provided $\Delta x<50 \mathrm{~mm}$ for $x / l<0.13$ and $\Delta x<100 \mathrm{~mm}$ for $x / l>0.13$. Therefore, to avoid probe interference, we acquired the 2-D correlation measurements using $\Delta z>8 \mathrm{~mm}$ for all $\Delta x$. Moreover, while acquiring the data, the mean velocities were continuously monitored to make sure the both the probes were located in the desired $y$ location.

\section{Results and discussion}

In this section, the measurements obtained from the pre-transitional region for the $h_{w}=$ 40, 60 and $80 \mathrm{~mm}$ cases are analysed to ascertain the initial disturbance growth and its associated transitional characteristics. 

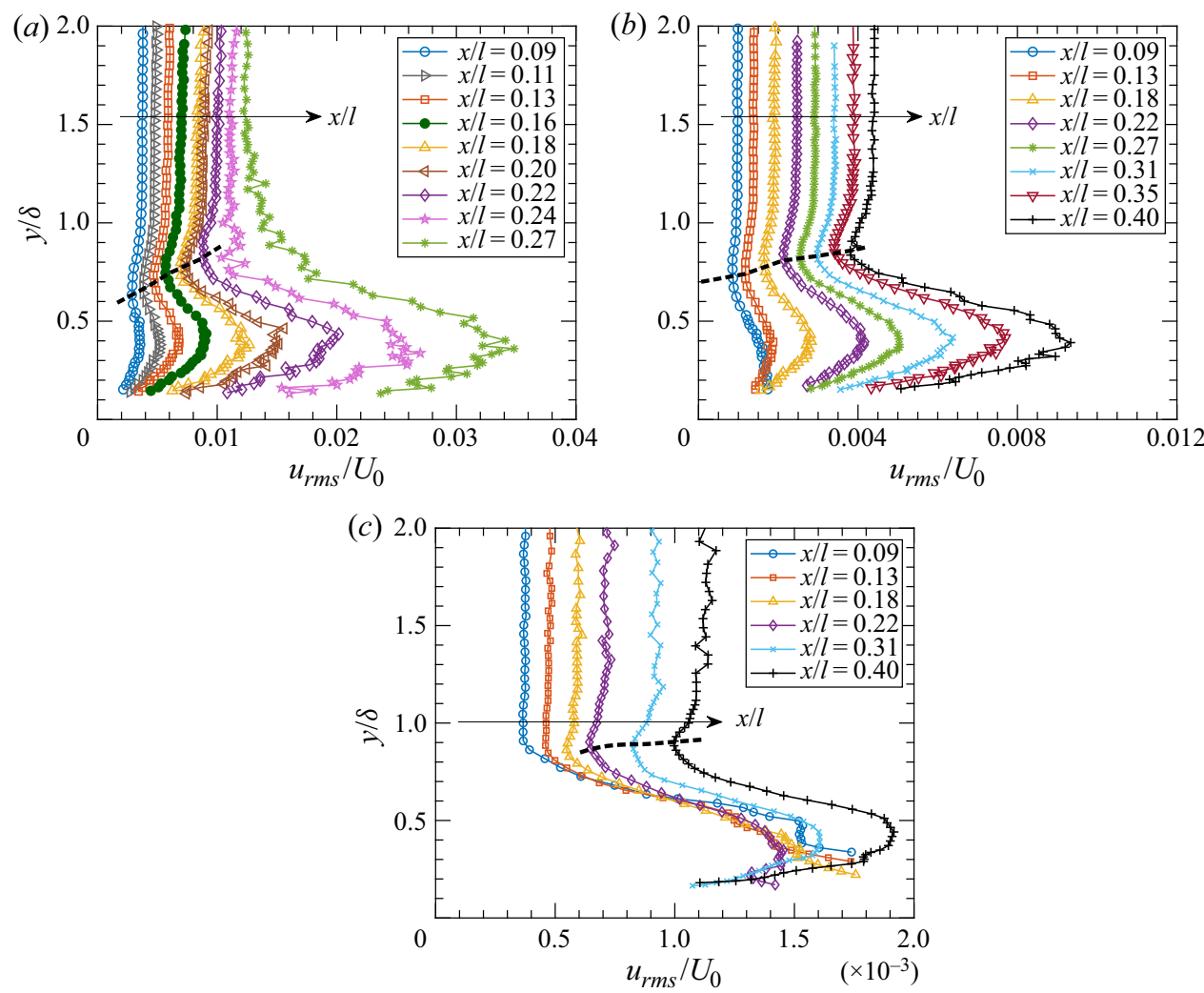

Figure 5. The $u_{r m s}$ profiles for the case $(a) h_{w}=40(b) h_{w}=60$ and (c) $h_{w}=80 \mathrm{~mm}$, with the outer minima connected with a dashed line. For $h_{w}=40 \mathrm{~mm}$, transition onset is at $x / l=0.24$.

\subsection{Disturbance profile and its growth}

The streamwise disturbance profiles $\left(u_{r m s}\right)$ at various downstream stations for $h_{w}=40,60$ and $80 \mathrm{~mm}$ are shown in figure 5 . Free-stream velocity $\left(U_{0}\right)$ and boundary layer thickness $(\delta)$ are used as normalising parameters. Two distinct observations can be made. Firstly, the magnitude of the turbulence intensity increases with downstream distance, including above the edge of the boundary layer $(y / \delta>1)$. It can be argued that, as the thickness of the wake and the boundary layer evolves in the downstream distance, the region of potential core between the wake and boundary layer decreases, resulting in a strengthening interaction between the wake and the boundary layer as the flow develops downstream. This is one of the important characteristics of the wake-boundary layer interaction problem, in contrast to the FST-induced transition case where the turbulence intensity above the boundary layer decreases in the downstream direction (Westin et al. 1994). Figure 5 also shows that the interaction occurs at a moderate level of turbulence intensity, which is discussed further later in this section.

The second observation is the presence of both a maximum and a local minimum disturbance amplitude inside the boundary layer $(y / \delta<1)$. The local minima for all $h_{w}=40,60$ and $80 \mathrm{~mm}$, lie above $y / \delta=0.7$ and shift towards the edge of the boundary layer further downstream, as indicated by the dashed lines in figure 5(a-c) respectively. Figure 5(a) shows that this local minimum persists until the point of transition onset $(x / l=0.24)$ and tends to disappear beyond that. For the case of $h_{w}=60 \mathrm{~mm}$ (figure $\left.5 b\right)$, 

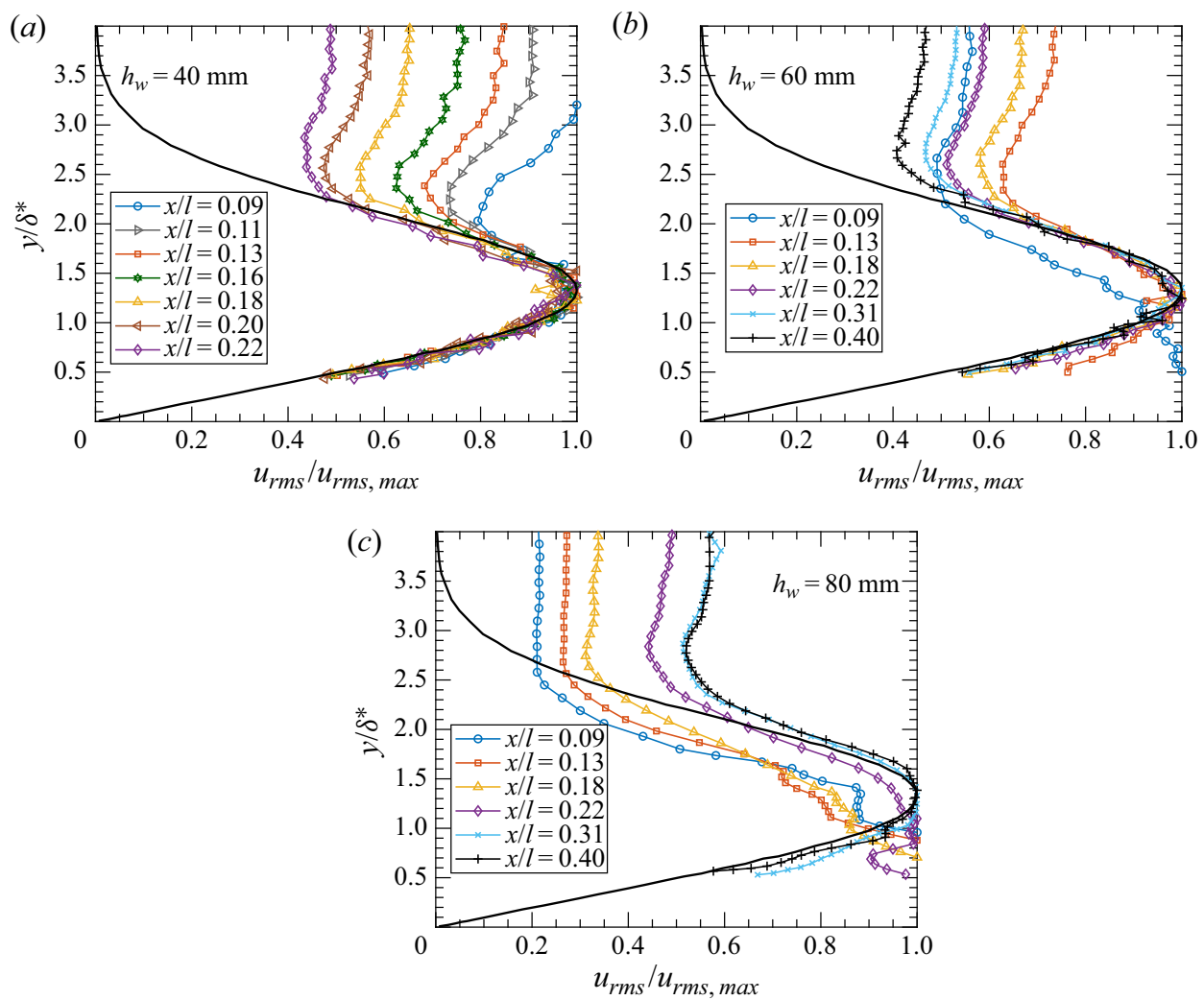

Figure 6. Normalised $u_{r m s}$ profiles for $(a) h_{w}=40,(b) h_{w}=60$ and (c) $h_{w}=80 \mathrm{~mm}$. The black solid line corresponds to transient growth theory, Luchini (2000).

the flow remains laminar throughout the domain and the minimum occurs at all measurement stations. On the other hand, for $h_{w}=80 \mathrm{~mm}$ (figure $5 \mathrm{c}$ ), a local minimum is noticed beyond $x / l=0.18$; moreover, the wall-normal profile also tends to change its shape beyond this station. A similar kind of behaviour was noticed for cylinder wake-induced transition by He et al. (2013), who ascribed this local minimum to the shear sheltering phenomenon (Jacobs \& Durbin 1998). Additional evidence for the presence of the shear sheltering phenomenon in the present experiment is provided by the spectral distributions (discussed later), where only low-frequency disturbances are found to be amplified below the location of the local minimum in $u_{r m s}$. Now considering the location of the maximum disturbance $\left(u_{r m s, \max }\right)$, this appears to remain constant at $y / \delta=0.4$ or $y / \delta^{*}=1.3$ for both cases; this is a characteristic shared by FST-induced bypass transition.

Figure 6(a) demonstrates that the disturbance profile in the pre-transitional region exhibits self-similarity if normalised by the local boundary layer displacement thickness $\left(\delta^{*}\right)$ and the local maximum disturbance $\left(u_{r m s, \max }\right)$. A number of the normalised profiles follow the profile predicted by the transient growth theory, developed by Andersson, Berggren \& Henningson (1999) and Luchini (2000), for $y<2 \delta^{*}$. This type of disturbance profile is generally observed in the presence of streamwise streaks or streamwise vortices (which are termed 'non-modal' disturbances, due to the absence of Tollmien-Schlichting, or T-S, waves). Cylinder wake-induced transition studies (Mandal \& Dey 2011; Ovchinnikov et al. 2006) and FST-induced bypass transition studies 

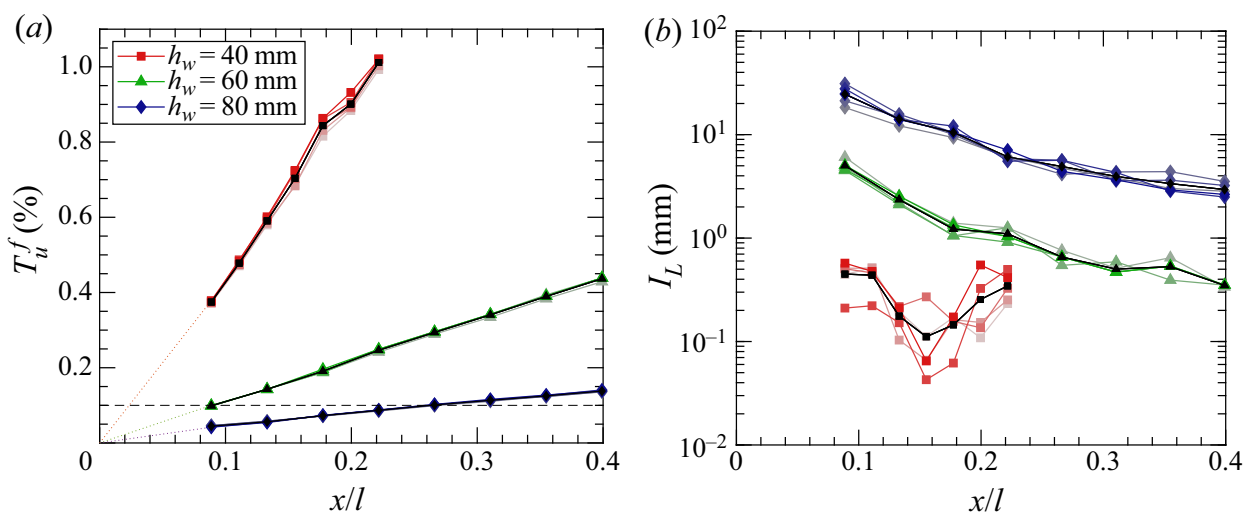

Figure 7. The black line with marker indicates the average $(a)$ forcing turbulent intensity, $T_{u}^{f}$ and $(b)$ the integral length scale, $I_{L}$ measured between $y / \delta \approx 1$ and 2 for $h_{w}=40,60$ and $80 \mathrm{~mm}$. The lighter to darker shades correspond to the measurements obtained between $y / \delta \approx 1$ and 2 .

(Westin et al. 1994; Matsubara \& Alfredsson 2001) also showed a similar non-modal distribution in the pre-transitional region.

However, the present results exhibit an additional characteristic when the aerofoil height is varied. In figure $6(a-c)$, the pre-transitional disturbance profiles are shown for the $h_{w}=40,60$ and $80 \mathrm{~mm}$ cases, respectively. It can be observed that the disturbance profile at the initial stage of the pre-transitional region does not always follow the non-modal distribution. Thus at $x / l=0.09$ for $h_{w}=60 \mathrm{~mm}$ and $x / l=0.13,0.18$ and 0.22 for $h_{w}=80 \mathrm{~mm}$ the location of $u_{r m s, \max }$ tends to occur closer to the wall than for the non-modal distribution of Luchini (2000) - in fact, more like a modal disturbance (see the spectral analysis and filtered $u_{r m s}$ profiles presented in figures 12 and 13 which provide greater detail). It can be reasoned that, if the aerofoil height is increased, then the initial interaction near the flat-plate leading edge occurs at a lower turbulence intensity and that, at some point, the non-modal forcing ceases to dominate. These results reveal the interesting transformation of the disturbance profile from modal type to non-modal type at these particular geometric and flow conditions, a hybrid behaviour not reported in any other experimental study in the literature. This arrangement, displaying such borderline transition characteristics, could therefore offer an opportunity to study the competing receptivity mechanisms in detail.

In FST-induced transition, whether the disturbance profile is modal (natural transition) or non-modal (bypass transition) is determined by the turbulence intensity level measured in front of the leading edge, since the disturbances propagate through the leading edge (Kosorygin \& Polyakov 1990). In the present wake-induced transition study, the turbulence propagating through the leading edge is smaller in magnitude $\left(T_{u}=0.04 \%\right.$ for $h_{w}=$ $40 \mathrm{~mm}$ and $0.03 \%-0.015 \%$ for $h_{w}=60$ and $80 \mathrm{~mm}$ ) when compared with the turbulence propagating through the boundary layer. As a consequence, the local turbulence intensity at the edge of the boundary layer is assumed to be the natural determining parameter. Obtaining $T_{u}$ at the edge of the boundary layer will be subjected to uncertainties in determining the exact location of boundary layer edge, which oscillates due to the unsteady low- and high-speed streaks inside the boundary layer. To minimise this uncertainty, we have used the average $T_{u}$ between $y / \delta=1$ and 2 as the forcing turbulence $\left(T_{u}^{f}\right)$. Moreover figure $7(a)$ shows that, in this region $(y / \delta=1$ to 2$)$, the variation in $T_{u}$ is not significant. 
Figure $7(a)$ exhibits that the forcing turbulence, $T_{u}^{f}$ increases linearly with the streamwise distance and that the growth rate decreases as the aerofoil is moved further away from the plate. By comparing the turbulent intensities in figure 7(a) with the corresponding disturbance profiles in figure 6 , it is inferred that, for $T_{u}^{f}>0.1 \%$, the disturbance profile possesses non-modal characteristics, whereas if $T_{u}^{f}<0.1 \%$, then the disturbance profile is more modal in character. This tallies with the case of FST-induced transition, where $T_{u}=0.1 \%$ is generally defined (Kosorygin \& Polyakov 1990; Fasel 2002) as the threshold between natural (T-S wave or modal disturbance) and bypass transition (non-modal disturbance, streaky structure). Despite tallying with the reported literature, $T_{u}^{f} \approx 0.1 \%$ cannot be defined as a criterion for modal to non-modal transformation in the present experiment, since the disturbance frequency is also influenced by the height of the aerofoil, discussed later. On the other hand, it is interesting to note that, as figure $7(a)$ reveals, $T_{u}^{f}$ increases linearly with streamwise distance while the wake and the boundary thicknesses increase as $x^{1 / 2}$ (Pope 2000), which invites further investigation. Figure $7(a)$ also illustrates that the various measured wake-boundary layer interactions occur at very different ranges of turbulence intensity: for $h_{w}=40 \mathrm{~mm}$ at moderate to high intensity $(0.35 \%-1.2 \%)$, for $h_{w}=60 \mathrm{~mm}$ at moderate intensity $(0.1 \%-0.4 \%)$ and for $h_{w}=80 \mathrm{~mm}$ at low $(<0.1 \%)$ to moderate intensity.

The integral length scale $\left(I_{L}\right)$ above the edge of the boundary layer is determined by multiplying the local mean velocity with the integral time scale obtained through autocorrelation. Figure $7(b)$ shows the streamwise variation of the average $I_{L}$ obtained between $y / \delta=1$ and 2 , showing that $I_{L}$ of the forcing turbulence varies little with height $y$ above the edge of the boundary layer, except for the $h_{w}=40 \mathrm{~mm}$ case. Moreover, $I_{L}$ decreases gradually in the downstream distance and generally seems to vary inversely with the $T_{u}$ shown in figure 7(a). It has been observed that, for isotropic turbulence (grid turbulence), turbulent intensity decreases and its corresponding length scale increases with the downstream distance (Fransson et al. 2005). Conversely, in the present forcing arrangement these trends are reversed, which may indicate that the wake turbulence interacting with the boundary layer is anisotropic. For $h_{w}=40 \mathrm{~mm}, I_{L}$ decreases by an order of magnitude or so up to $x / l=0.18$ and subsequently increases by the same order: this, and the increased sensitivity to $y$ location, may be the consequence of strong wake interaction and transition onset occurring at $x / l=0.24$.

From figure $8(a)$, it can be seen that, in the pre-transitional region, maximum energy $\left(u_{r \text { ms } \text { max }}^{2}\right)$ growth is exponential in the streamwise direction, matching well with the empirical fits $e^{25.7(x / l)}, e^{9.6(x / l)}$ and $e^{3.8(x / l)}$ for $h_{w}=40 \mathrm{~mm}, 60 \mathrm{~mm}$ and $80 \mathrm{~mm}$, respectively. This difference can presumably be attributed to the variation in $T_{u}^{f}$ with aerofoil height: Fransson et al. (2005) and Norimatsu, Takai \& Matsubara (2011) have shown that, for the case FST-induced bypass transition, if FST levels are increased, the energy growth rate also increases. However, the extended streamwise region of exponential growth contrasts with the characteristics of cylinder wake-induced transition (Mandal \& Dey 2011; He et al. 2013) and FST induced-transition (Fransson et al. 2005), where the disturbance energy grows exponentially only for a short initial region, later growing algebraically. Figure $8(b)$ demonstrates the streamwise growth of the integrated boundary layer energy. It is calculated by using (3.1), and the integration is carried out up to the boundary layer thickness. The solid markers in figure $8(b)$ correspond to the point where non-modal disturbance profiles are observed. For $h_{w}=40 \mathrm{~mm}$ the total energy increases exponentially in both the pre-transitional (solid marker) and transitional regimes (hollow marker). On the other hand, for $h_{w}=60$ and $80 \mathrm{~mm}$ in figure $8(a, b)$, a few of the 

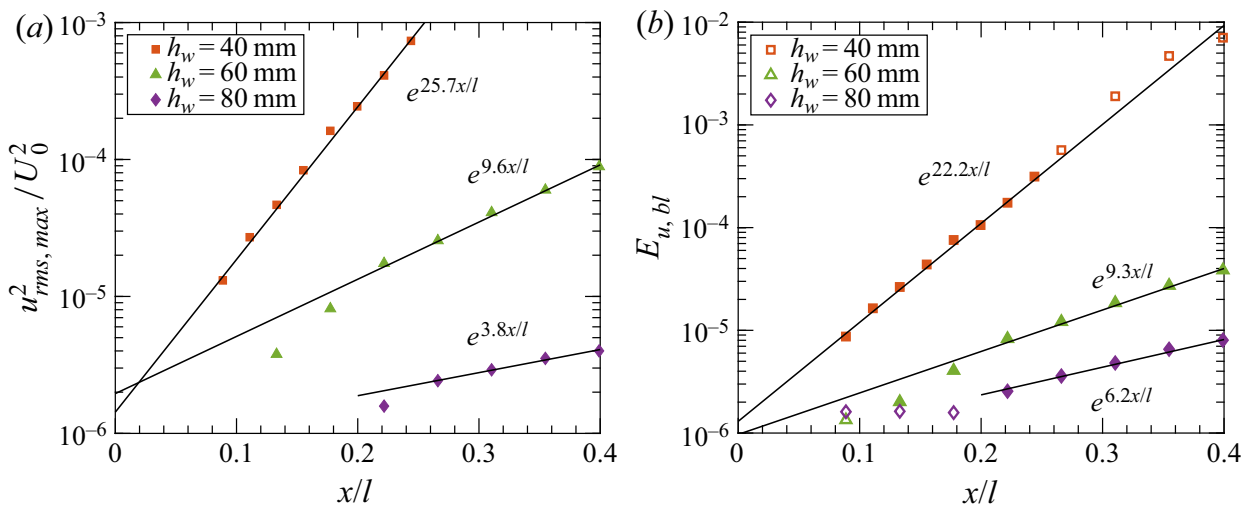

Figure 8. Streamwise growth of the (a) maximum $u$-disturbance energy and $(b)$ integral energy inside the boundary layer.

measurements deviate from the exponential fit, and this corresponds to the transformation from modal to non-modal regime.

$$
E_{u, b l}=\int_{0}^{\delta} u_{r m s}^{2} \mathrm{~d} y /\left(U_{0}^{2} \delta\right) .
$$

In both the cylinder wake and FST cases, the literature attributes the short region of exponential growth to the receptivity process. In the case of a cylinder wake, He et al. (2013) showed through dynamic mode decomposition that the shedding frequency of the cylinder wake penetrated directly into the boundary layer, producing a short, initial region of exponential energy growth; the subsequent algebraic stage of the growth was attributed to the destabilisation of the secondary vortex. In the present experiment, the absence of vortex shedding seems to rule out this mechanism. For FST-induced bypass transition, Fransson et al. (2005) attributed the region of short exponential growth to the free-stream disturbances adjusting to the boundary layer. Such an explanation might fit the present results if the increasing intensity of forcing with downstream distance had the effect of distributing the 'adjustment' process - and hence the exponential growth characteristic - throughout the boundary layer, rather than near the leading edge as in FST-induced transition.

\subsection{Power spectral density}

Further insight into the influence of the wake disturbances on the boundary layer is provided by the power spectral density (PSD) of the streamwise perturbation. Firstly, the spectral characteristics in the wake region are demonstrated by the 2-D spectral contour plots obtained at three different $x / l$ for $h_{w}=40,60$ and $80 \mathrm{~mm}$, shown in figure 9 . Here, both the spectral power $(p)$ and the frequency $(f)$ are normalised in a way that, $F=2 \pi f v \times 10^{6} / U_{0}^{2}$ and $P_{n}=p / 0.5 U_{0}^{2} \Delta F$, where $v$ is the kinematic viscosity. The black dashed line in figure 9 indicates the maximum amplitude of the PSD at various $y / \delta$, which can be used as an indicator for the peak forcing frequency $\left(F_{e}\right)$ from the wake. The blue dashed line indicates the boundary layer thickness. It is to be noted that, for $h_{w}=40 \mathrm{~mm}$, the spectrum is plotted from the wall up to the wake core and for $h_{w}=60$ and $80 \mathrm{~mm}$, the spectrum is plotted up to a point slightly below the wake core.

From figure 9, it can be inferred that, for all the three cases, a broad bandwidth of frequency from the wake is filtered down as it approaches the boundary layer. As noticed 

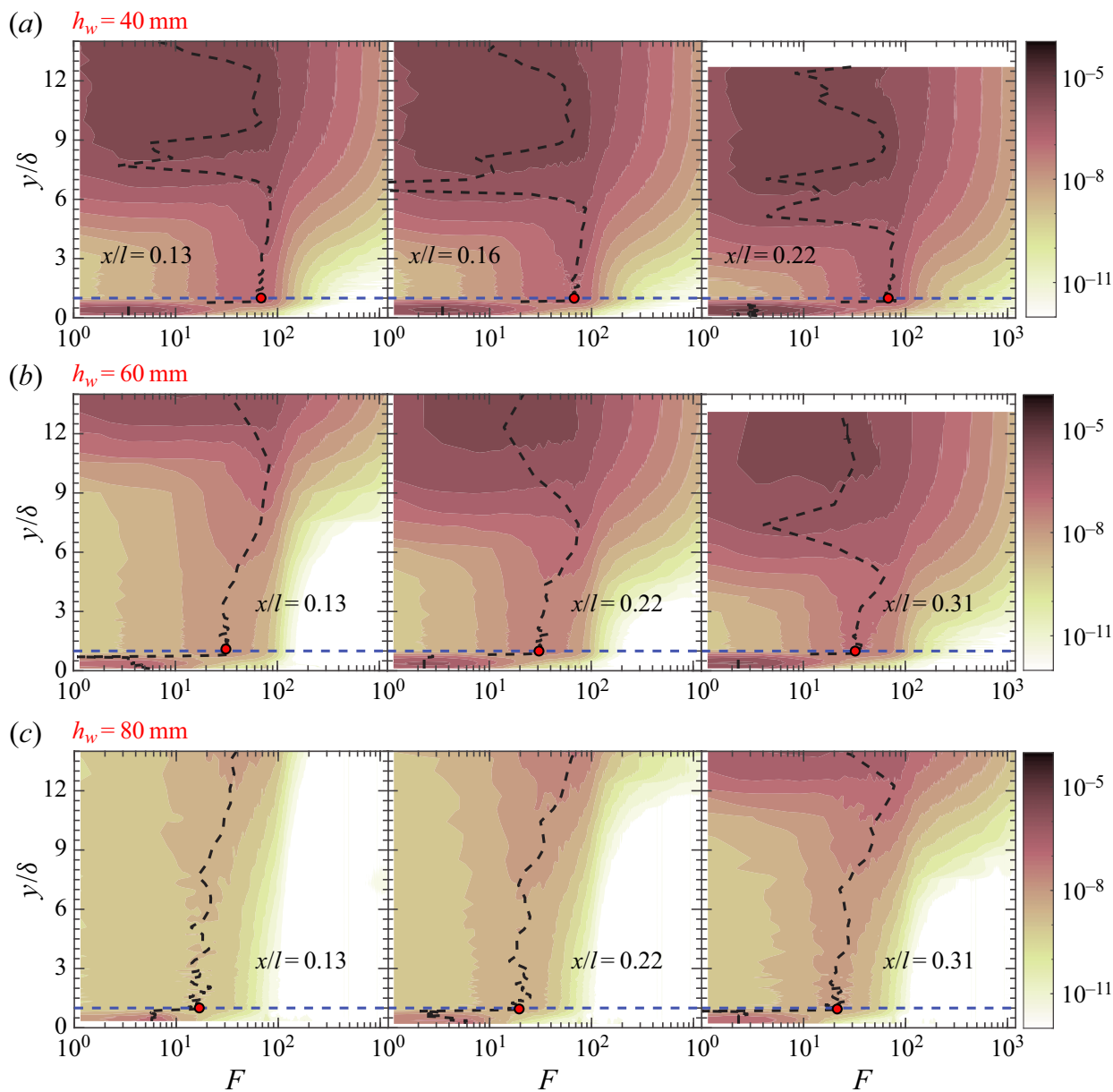

Figure 9. Two-dimensional contours of the PSD obtained for three different $h_{w}$ at various streamwise stations. The black dashed line indicates the maximum amplitude of the PSD, and the blue dashed line indicates the boundary layer thickness.

in the previous sections, the wake forcing outside the boundary layer is continuous and increasing in intensity with downstream distance, but figure 9 reveals that the forcing frequency does not change with streamwise location for all the three cases. In particular, for a given $h_{w}, F_{e}$ on the boundary layer edge (marked with red dot) does not vary with $x / l$, but $F_{e}$ decreases with increasing $h_{w}$. The frequency value, $F_{e}$ for various $x / l$ values remains constant at $\approx 70,30$ and 20 for the $h_{w}=40 \mathrm{~mm}, 60 \mathrm{~mm}$ and $80 \mathrm{~mm}$ respectively. This is one of the interesting characteristics of the aerofoil wake-boundary layer interaction observed in the present study. This observation can be seen from the 1-D PSD as plotted in figure 10, where the forcing frequency spectrum is dependent on the height (figure 10a), but largely independent of the streamwise station in the pre-transitional zone (figure 10b). These observations are similar to the case of circular cylinder wake interaction where the shedding frequency decreases with increase in the cylinder height (He et al. 2016), although there is no discrete vortex shedding in the current study.

The influence of the wake disturbances inside the boundary layer is provided by the 1-D PSD at different wall-normal stations. Figure 11 presents the spectra for the $h_{w}=40$, 

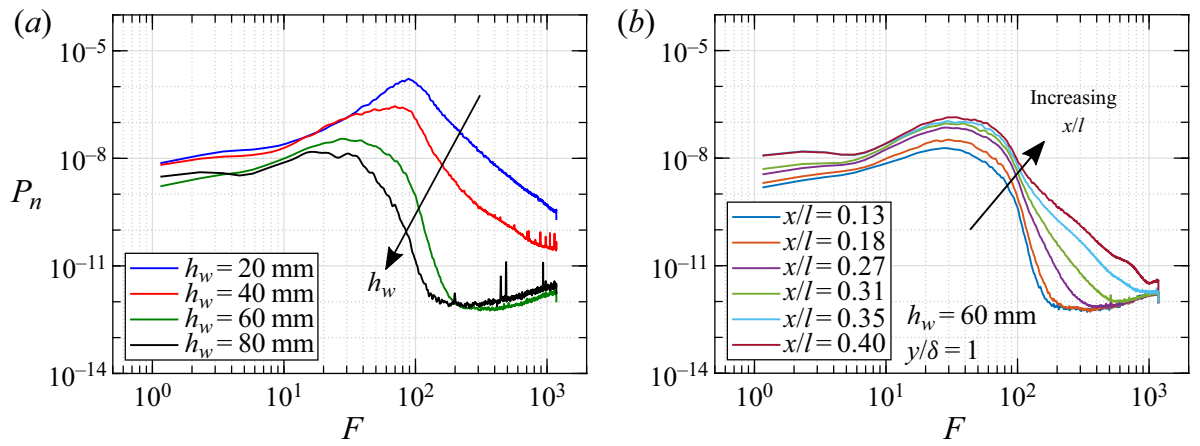

Figure 10. Power spectral density, obtained at the edge of the boundary layer for $(a)$ various heights of the aerofoil and $(b)$ various streamwise stations for $h_{w}=60 \mathrm{~mm}$. Here, $x / l$ correspond to the $h_{w}$ in figure $10(a)$ is $0.04,0.18,0.18$ and 0.35 respectively.
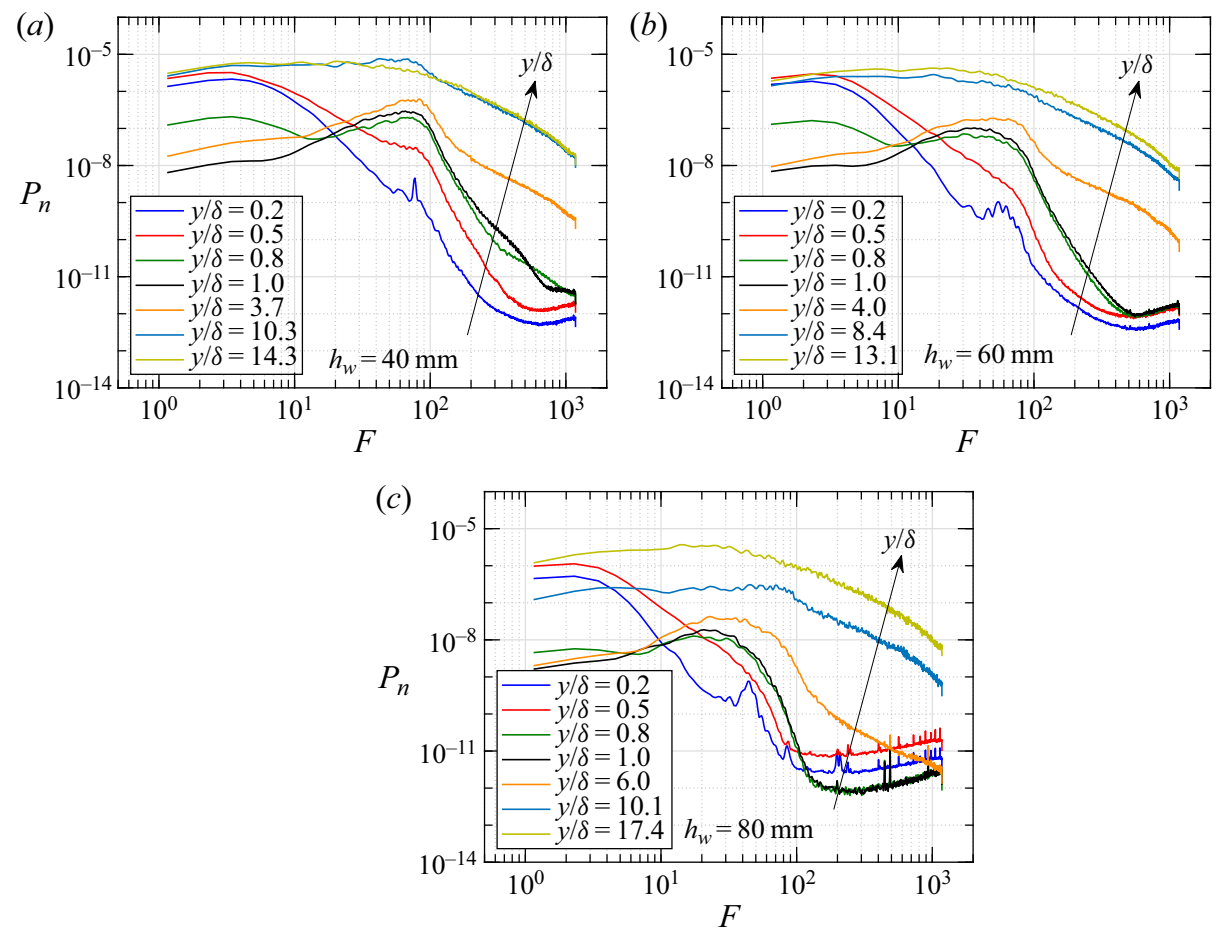

Figure 11. Normalised PSD of the streamwise perturbations at various wall-normal stations for $(a) x / l=0.16$, $h_{w}=40(b) x / l=0.31, h_{w}=60 \mathrm{~mm}$ and (c) $x / l=0.4, h_{w}=80 \mathrm{~mm}$. The maximum value of $y / \delta$ in all three panels is approximately close to the wake centreline. For reference, respective $\delta$ and $\delta^{*}$ for (a) 2.75 and 0.87 , (b) 3.8 and 1.17, (c) 4.3 and 1.4.

60 and $80 \mathrm{~mm}$ cases at $x / l=0.16,0.31$ and 0.4 , respectively. In this figure, the wake centreline lies close to $y / \delta=14.3$ for $h_{w}=40 \mathrm{~mm}, y / \delta=13.1$ for $h_{w}=60 \mathrm{~mm}$ and $y / \delta \approx 15$ for $h_{w}=80 \mathrm{~mm}$. In the region of the potential flow core $(y / \delta=3.7,4$ and 6 for $h_{w}=40 \mathrm{~mm}, 60 \mathrm{~mm}$ and $80 \mathrm{~mm}$ respectively), the spectral power is intensified over a band of mid-range frequencies $(F=30-110)$. Furthermore, as the edge of the boundary layer is approached $(y / \delta=1)$, high frequency perturbations associated with small-scale 
Transition induced by the upstream aerofoil wake
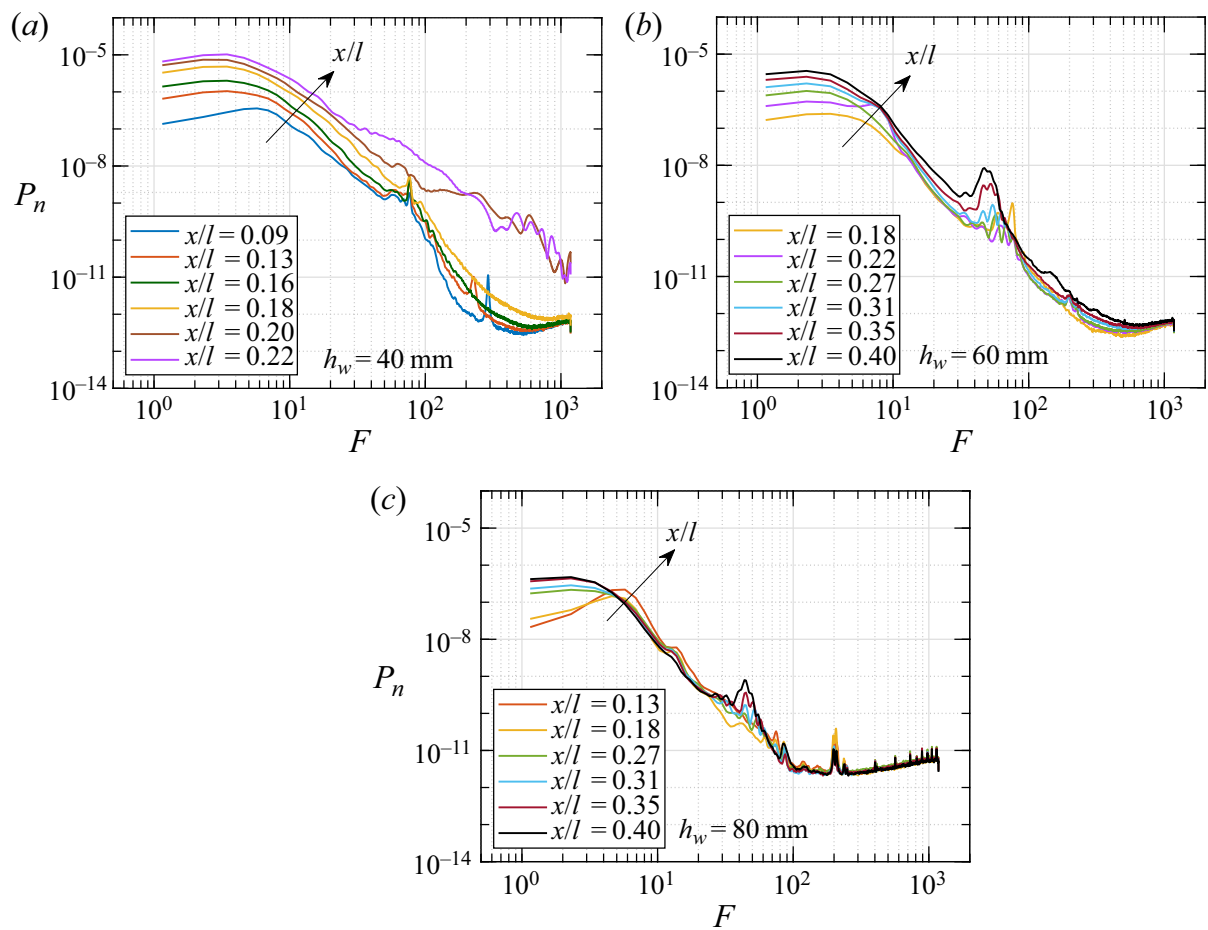

Figure 12. Normalised PSD for various streamwise stations at $y / \delta=0.2\left(y / \delta^{*} \approx 0.6\right)$ for the case $(a)$ $h_{w}=40 \mathrm{~mm},(b) 60 \mathrm{~mm}$ and (c) $h_{w}=80 \mathrm{~mm}$.

turbulence in the wake are attenuated, while the bandwidth of mid-range frequency still persists. Inside the boundary layer $(y / \delta=0.8,0.5$ and 0.2$)$, only the low-frequency $(F=1-30)$ perturbations are intensified, while the others are attenuated. This can be attributed to the shear sheltering phenomenon (Jacobs \& Durbin 1998). This effect occurs close to the location of local minimum of $u_{r m s}$ (dashed line in figure 5), supporting the association observed by He et al. (2013), as mentioned earlier.

The spectra at $y / \delta=0.2$ in figure 11 show that, for all the aerofoil heights considered, $h_{w}=40,60$ and $80 \mathrm{~mm}$, a small group of mid-range frequencies are amplified. The disturbances in this part of the boundary layer are further explored in figure 12, where the spectra at $y / \delta=0.2$ for various streamwise stations are shown. It can be seen that the spectral power around $F=40-80$ is intensified at all streamwise stations, and that, for $h_{w}=60$ and $80 \mathrm{~mm}$, this amplification becomes more pronounced downstream with an accompanying reduction in frequency. This characteristic is typical of the evolution of T-S waves. As a side note, high-frequency content can be seen at $x / l=0.2$ and 0.22 for the $h_{w}=40 \mathrm{~mm}$ case, which probably a sign of the emergence of turbulent spots (Blair 1992).

To ascertain the nature of these amplified disturbances, the $u_{r m s}$ distribution for selected frequencies are plotted in figure 13. It shows, for all the $h_{w}$, the $u_{r m s}$ profiles for $F \leq 21$ reach a maximum roughly at the middle of the boundary layer, $y / \delta^{*}=1.3$, as is typical of non-modal disturbances. Conversely, at $F=78$ for $h_{w}=40 \mathrm{~mm}, F=52$ for $h_{w}=$ $60 \mathrm{~mm}$ and at $F=44$ for $h_{w}=80 \mathrm{~mm}$ (the mid-range-frequency peaks in figure 12), the disturbances have peak amplitude in the near-wall region $\left(y / \delta^{*}=0.5-0.7\right)$ which is typical of T-S waves. Filtered $u_{r m s}$ profiles corresponding to amplified mid-range frequencies observed in the near-wall spectrum (figure $12 a-c$, table 2) are plotted in figure 14. It can 

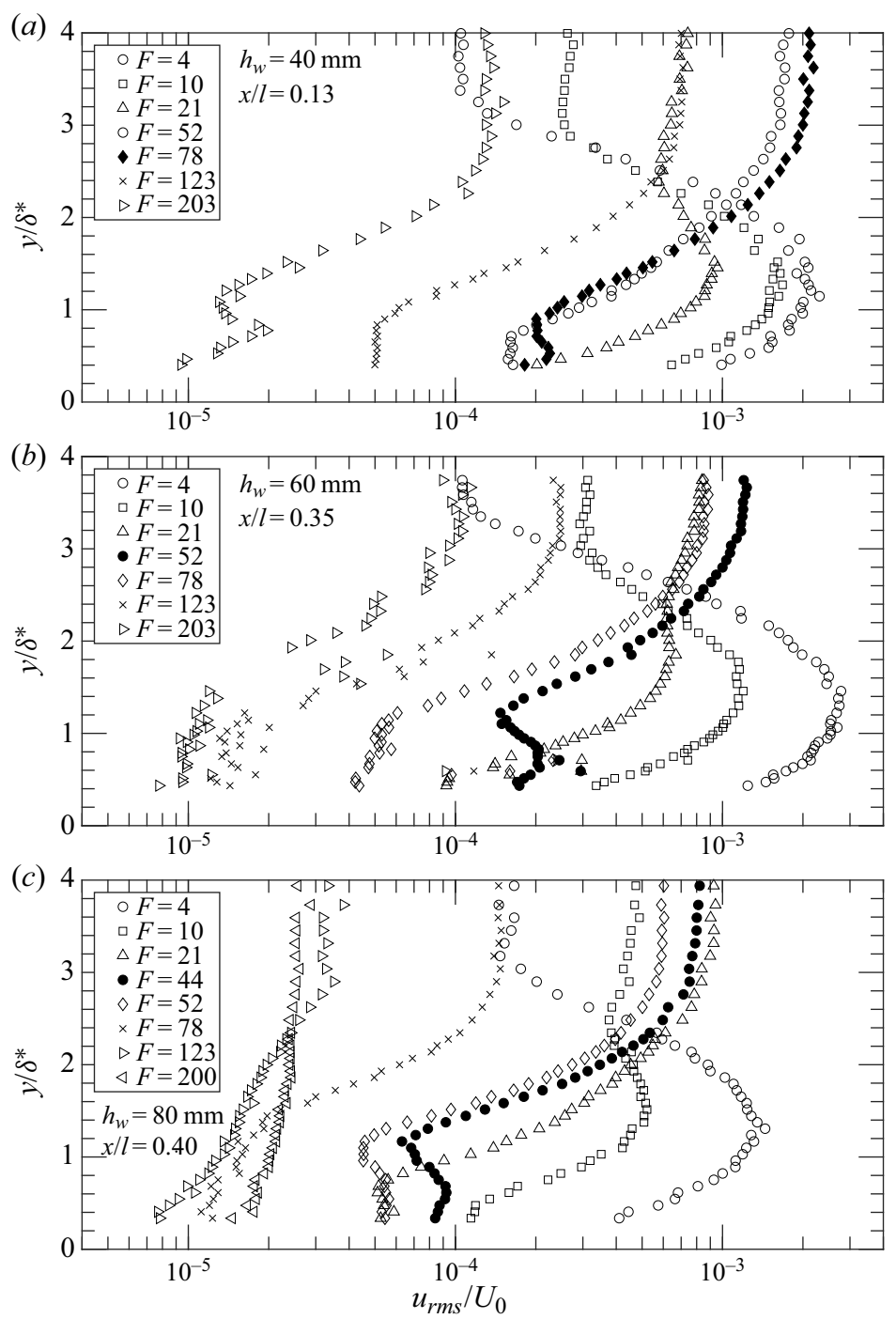

Figure 13. The $U_{r m s}$ distributions obtained for selected frequencies at $x / l=0.13$ for $h_{w}=40 \mathrm{~mm}$, $x / l=0.35$ for $h_{w}=60 \mathrm{~mm}$ and at $x / l=0.4$ for $h_{w}=80 \mathrm{~mm}$.

be seen that the local maximum at the near-wall region $\left(y / \delta^{*}=0.5-0.7\right)$ increases in amplitude with streamwise distance for $h_{w}=60$ and $80 \mathrm{~mm}$; figure 15(a) (solid marker) shows that the amplification is exponential. On the other hand, for $h_{w}=40 \mathrm{~mm}$, the filtered $u_{r m s}$ profile does not vary with the streamwise station despite the presence of a near-wall peak. The reason for the lack of spatial evolution for the $h_{w}=40 \mathrm{~mm}$ case is discussed in the next section (stability analysis).

It seems evident from the characteristics in figures $12(a-c), 13,14$ and $15(a)$, that the amplification seen at mid-range frequencies and its exponential energy growth is due to the T-S waves and that both modal and non-modal disturbances coexist and are amplified in the present experiment. This coexistence can be attributed to the wake-boundary layer interaction occurring at the moderate level of turbulence: Kosorygin \& Polyakov (1990) and Kenchi, Matsubara \& Ikeda (2008) have shown, for a moderate level of FST-induced 


\begin{tabular}{ccccc}
$\begin{array}{l}h_{w} \\
(\mathrm{~mm})\end{array}$ & $\begin{array}{c}x \\
(\mathrm{~mm})\end{array}$ & $x / l$ & $\begin{array}{c}F \\
\text { (computed) }\end{array}$ & $\begin{array}{c}F \\
\text { (measured) }\end{array}$ \\
\hline \multirow{3}{*}{40} & 200 & 0.09 & 126 & 77 \\
& 250 & 0.11 & 109 & 77 \\
& 300 & 0.13 & 100 & 77 \\
& 350 & 0.16 & 89 & 77 \\
60 & 400 & 0.18 & 81 & 77 \\
& 500 & 0.22 & 70 & 61 \\
& 600 & 0.27 & 62 & 56 \\
& 700 & 0.31 & 56 & 53 \\
& 800 & 0.35 & 50 & 52 \\
& 900 & 0.4 & 48 & 47 \\
& 600 & 0.27 & 62 & 54 \\
& 700 & 0.31 & 56 & 47 \\
& 800 & 0.35 & 50 & 45 \\
& 900 & 0.4 & 47 & 44
\end{tabular}

Table 2. Comparison of the frequencies from the $N$-factor (figure $16 b$ ) with the frequencies corresponding to the spatial amplification in figure 12 . Here, $F$ in the stability calculation is defined as, $F=\omega / R e_{\delta^{*}}$.

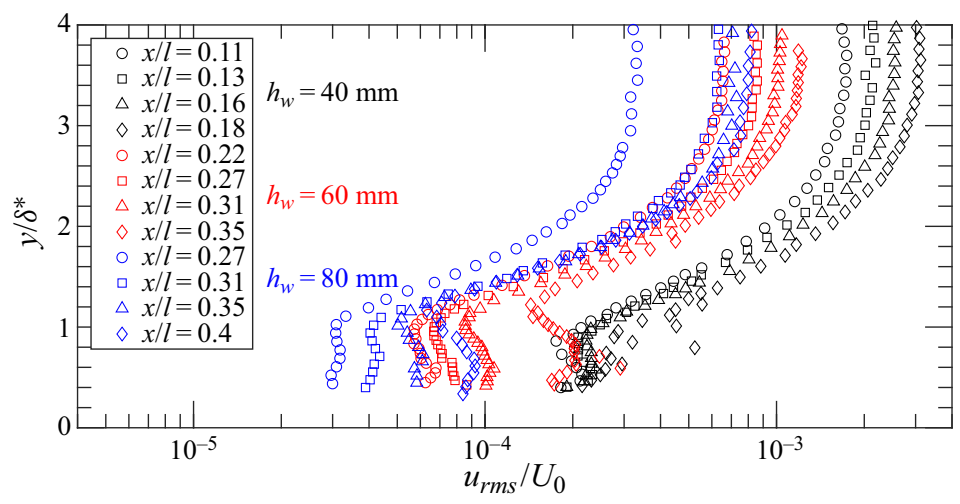

Figure 14. The $u_{r m s}$ profiles obtained for the measured mid-range frequencies (listed in table 2) observed in the near-wall spectrum (figure 12) for $h_{w}=40,60$ and $80 \mathrm{~mm}$.

transition, that a T-S wave coexists with the non-modal $u_{r m s}$ distribution. However, other characteristics such as maximum energy growth and the spacing of streaky structures (discussed later) differ from the present results.

Figure 12 also shows higher-frequency spikes around $F=300$ and 200 for $h_{w}=40 \mathrm{~mm}$ $(x / l=0.09$ and 0.13$)$ and $F=200$ for $h_{w}=60$ and $80 \mathrm{~mm}$, albeit at very low amplitudes. The corresponding $u_{r m s}$ distributions in figure 13 show a secondary, near-wall peak for $h_{w}=40 \mathrm{~mm}$ and not for $h_{w}=60$ and $80 \mathrm{~mm}$. The origin of these high-frequency disturbances is unknown.

To study the influence of modal characteristics on the non-modal $u_{r m s}$ profiles (figure 6), a band-stop filter is applied to the raw signals of $h_{w}=40$ and $60 \mathrm{~mm}$, with a bandwidth of $10 \mathrm{~Hz}$ centred at mid-range frequencies. The band-stop filter leaves most frequencies unaltered, except the frequencies in the specified range whose magnitude will be attenuated. Here, the signals corresponding to the mid-range frequencies are attenuated, and the $u_{r m s}$ profiles and the streamwise growth of the associated peak kinetic 

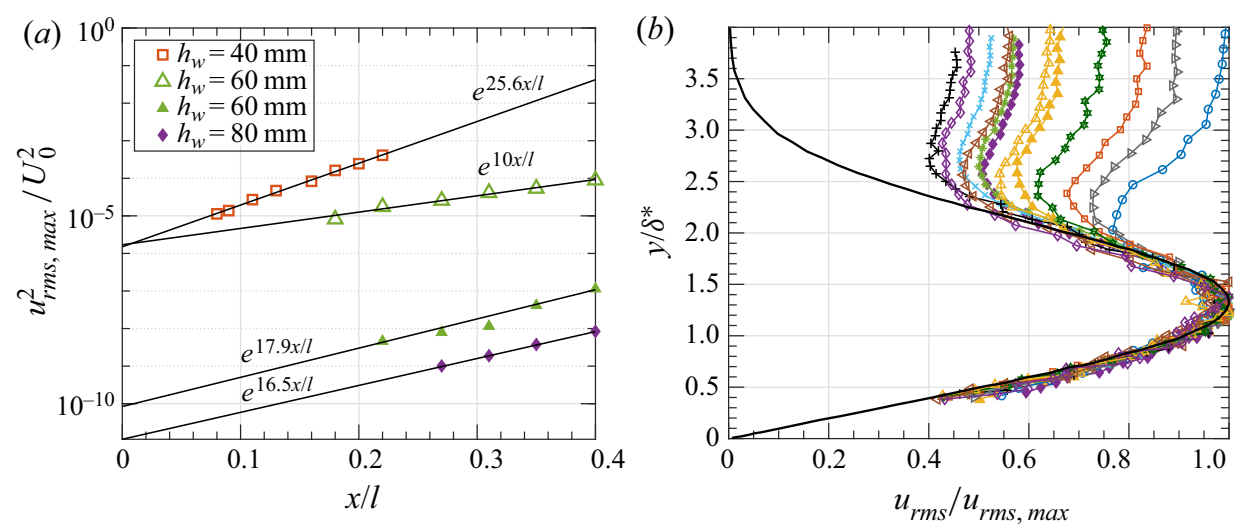

Figure 15. (a) Streamwise energy growth of the local $u_{r m s, m a x}$ extracted from the filtered profiles. Solid marker: near-wall $u_{r m s, \max }$ corresponding to the mid-range frequencies in figure 14. Hollow marker: $u_{r m s, \max }$ from the band-stop filtered (attenuating the mid-range frequencies) profiles. (b) Normalised $u_{r m s}$ profiles after applying the band-stop filter to the raw signals of $h_{w}=40 \mathrm{~mm}$ (hollow markers) and $60 \mathrm{~mm}$ (solid markers), marker legends are similar to figure 6 .

energy are obtained, as shown in figure 15 . The band-stop filtered $u_{r m s}$ profiles follow the non-modal distribution (figure 15b), and the kinetic energy growth is exponential, see the hollow markers in figure $15(a)$. Moreover, the growth rates $\left(e^{25.6}\right.$ and $\left.e^{10}\right)$ closely follow the unfiltered growth shown in figure $8(a)$. This technique was repeated for multiple bandwidths up to $120 \mathrm{~Hz}$, all centred at the mid-range frequency, and the variation in the results was insignificant. This analysis indicates that the disturbance energy corresponding to the non-modal or modal disturbance (referring to hollow and solid markers in figure 15a), or their combined effect (figure $8 a$ ), evolves exponentially in the streamwise distance. At the same time, while comparing the growth rates of $h_{w}=60 \mathrm{~mm}$ in figure 15(a) (hollow and solid markers), it appears that the modal disturbance evolves faster than the non-modal disturbance.

The influence of mixed disturbance characteristics (both modal and non-modal) on the laminar breakdown process is unknown. From the literature, it is generally observed that the laminar breakdown occurs when $u_{r m s} / U_{0}>1.5 \%$ in the near-wall region for natural transition (Fasel 2002) and $u_{r m s} / U_{0}>10 \%$ for FST-induced bypass transition (Westin et al. 1994; Balamurugan \& Mandal 2017). However, in the present study, for $h_{w}=40 \mathrm{~mm}$, $u_{r m s} / U_{0}$ corresponding to the modal disturbance (figure 14 ) is approximately $0.1 \%$ and, for the non-modal disturbance(figure $5 a$ ), approximately $2 \%$. Neither modal nor non-modal disturbances reach the suggested threshold amplitudes on their own and it may be that a combination of these two mechanisms is responsible for breakdown in some of the current experiments. This is identified later as an area for further research.

\subsection{Stability analysis}

Spatial linear stability analysis has been carried out on the measured wake-boundary layer profile. The aim of this numerical analysis is to verify whether the spatial amplification of the modal disturbances observed in the near-wall region (for $h_{w}=60 \mathrm{~mm}$ ) is consistent with the presence of T-S waves. To compute the unstable modes, the algorithm of Haj-Hariri (1988), Varghese (2016) and Mitra (2018) was employed with a Chebyshev spectral method (Schmid \& Henningson 2012) for discretising the equation in the wall-normal direction. The method was validated with the more relevant study conducted 

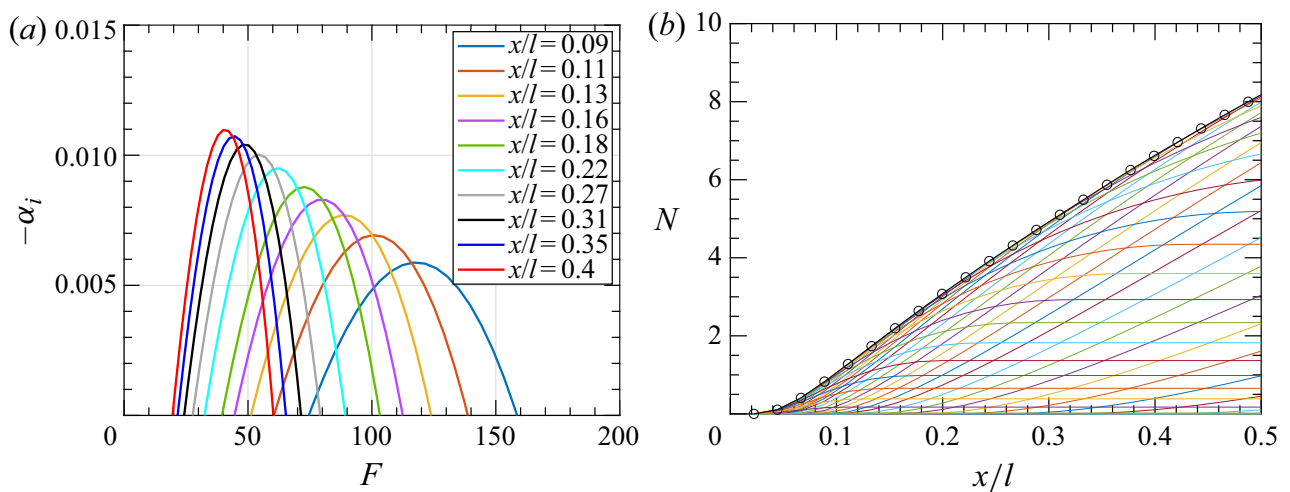

Figure 16. (a) Growth rate and (b) $N$ factor of the boundary layer mode from the wake-boundary layer profile for the case $h_{w}=60 \mathrm{~mm}$. In $(b)$, solid lines correspond to $n$ values, and the line with markers indicates the envelope of $n$ values, i.e. $N$ factor.

by Liou \& Liu (2001), see Appendix A. To obtain a smooth base flow profile without any discontinuities in the derivatives, a confluent wake-boundary layer profile is generated numerically to match the measured wake profile. Appendix B describes the methodology utilised to arrive a smooth base flow profile.

By solving the Orr-Sommerfeld equation with the obtained base flow velocity profiles three different unstable modes are noted: one boundary layer mode and two wake modes. Boundary layer modes are more relevant to the present measurements at $h_{w}=60 \mathrm{~mm}$, therefore the spatial growth rate $\left(-\alpha_{i}\right)$ of the unstable boundary layer mode is obtained as shown in figure 16(a). It can be observed that the maximum growth rate increases in the downstream direction, while its corresponding frequency decreases. The same trends were previously noted (figure $12 b, c$ ) for the mid-range frequency of the streamwise spectral distribution.

It is worth mentioning that the growth rates of the boundary layer mode shown in figure $16(a)$ were found to be same for the $h_{w}=40$ and $80 \mathrm{~mm}$ base flow velocity profiles, and it is understood from Liou \& Liu (2001) that, above a certain $h_{w}\left(>10 \delta^{*}\right)$, the boundary layer mode is unaffected, i.e. eigenvalues remains constant. In the present experiment (for $h_{w}=40,60$ and $80 \mathrm{~mm}$ ), the aerofoil was positioned at $>28 \delta^{*}$ for all streamwise stations, which is significantly higher than this threshold. Therefore, the similar growth rate for the $h_{w}=40,60$ and $80 \mathrm{~mm}$ base flow velocity profiles is consistent with the findings of Liou \& Liu (2001).

To compare the mid-range frequencies observed in the spectrum (figure 12a-c) with those of the modal disturbances (T-S waves), the $N$-factor of the boundary layer mode is calculated. The $N$-factor or $e^{N}$ technique is a method based on linear stability theory to predict the transition location and it was first proposed by Smith \& Gamberoni (1956) and Van Ingen (1956). Here, $N$ is defined as the maximum amplitude factor of the disturbance, which is obtained by integrating the unstable modes $\left(n=\int_{x_{0}}^{x}-\alpha_{i} \mathrm{~d} x\right)$ from $x_{0}$ to $x$ for a given $\omega$ (angular frequency); $x_{0}$ is usually defined as the station where the flow becomes first unstable, but the method can be applied to determine the logarithmic gain between any two stations. By calculating the $n$ for range of $\omega$, a set of $n$-curves is obtained, shown as a solid line in figure $16(b)$ and the envelope of the $n$-curves gives the maximum amplification factor $(N)$. It can be hypothesised that, if the frequency of the most amplified boundary layer mode (from figure 16b) is comparable to that of the spatially amplified, mid-range 
frequencies prominent in figure $12(b)$, then it would be a strong indication that T-S waves are present in the current experiment. Table 2 provides the comparison between the two frequencies for various streamwise stations.

Table 2 shows that, for $h_{w}=60$ and $80 \mathrm{~mm}$, the computed frequencies for the most-amplified modal disturbances, analysed in the presence of the wake defect, are comparable to those measured in the present experiment (figure 12b,c), and both exhibit the same trend with downstream distance. The small differences may be associated with slight mismatches between boundary layer profiles measured in the experiment and used for the computation. On the other hand, for $h_{w}=40 \mathrm{~mm}$, the measured frequencies, which remain constant with streamwise distance, are significantly lower than the computed frequencies, particularly for the more upstream stations. These results, along with the growth rates discussed earlier, suggest that the boundary layer response is a trade-off between (a) an improving frequency match (between forcing and the naturally most amplified frequencies) as $h_{w}$ increases and $(b)$ an increasing forcing amplitude as $h_{w}$ decreases. Further, the non-spatial evolution of the filtered $u_{r m s}$ profile in $h_{w}=40 \mathrm{~mm}$ (figure 14) is consistent with the mismatch between the measured mid-range frequencies and the computed, naturally most amplified frequencies. Nevertheless, the filtered $u_{r m s}$ profiles of all the measured mid-range frequencies exhibit a near-wall peak which matches reasonably closely the computed eigenfunctions (solid lines) shown in figure 17. These eigenfunctions were obtained for the $x / l$ station where mid-range frequencies are most prominent in the spectrum for $h_{w}=40,60$ and $80 \mathrm{~mm}$. The agreement between the stability calculations and the measurements supports our view that T-S waves are likely to be present. Further, this observation leads to the hypothesis that, if a particular disturbance frequency from the passing wake penetrates through the boundary layer, and if that corresponds to a significantly amplified mode, then spatial amplification of the near-wall peak will be observed.

\subsection{Spanwise correlation}

For the cases where the disturbance profile displayed non-modal characteristics typical of bypass transition, the existence of longitudinal streaky structures was investigated by means of two-point spatial correlation measurements. Both streamwise and spanwise correlations were conducted, By following equation (3.2), for the streamwise correlation measurement, one hot-wire probe was fixed at a given station and the location of the second probe was varied in both streamwise $(\Delta x)$ and spanwise $(\Delta z)$ senses; for the spanwise correlation measurement, the second probe was moved only in the spanwise sense. The probe mounting techniques and the limitations of the spatial correlation measurements are briefly discussed in $\S 2.2$

$$
\begin{aligned}
R & =\frac{\overline{u(t, x, z) u(t, x+\Delta x, z+\Delta z)}}{u(x, z)_{r m s} u(x+\Delta x, z+\Delta z)_{r m s}}, \\
R_{t} & =\frac{\overline{u(t, x, z) u(t+\Delta t, x+\Delta x, z+\Delta z)}}{u(x, z)_{r m s} u(x+\Delta x, z+\Delta z)_{r m s}} .
\end{aligned}
$$

Here, $\Delta t$ is obtained using Taylor's hypothesis, so $\Delta t=\Delta x / \bar{U}$ (streamwise probe separation/local mean velocity), a method predominantly used in identifying the coherent structures in turbulent flows (Dixit \& Ramesh 2010). Figure 18(a) shows the contours of the streamwise correlation function obtained, following the procedure described above, for the $h_{w}=40 \mathrm{~mm}$ case. For this measurement, the fixed probe was located at $x / l=$ $0.13, y / \delta^{*}=1.3$ and $z=0$. The second hot-wire probe was traversed at the same 

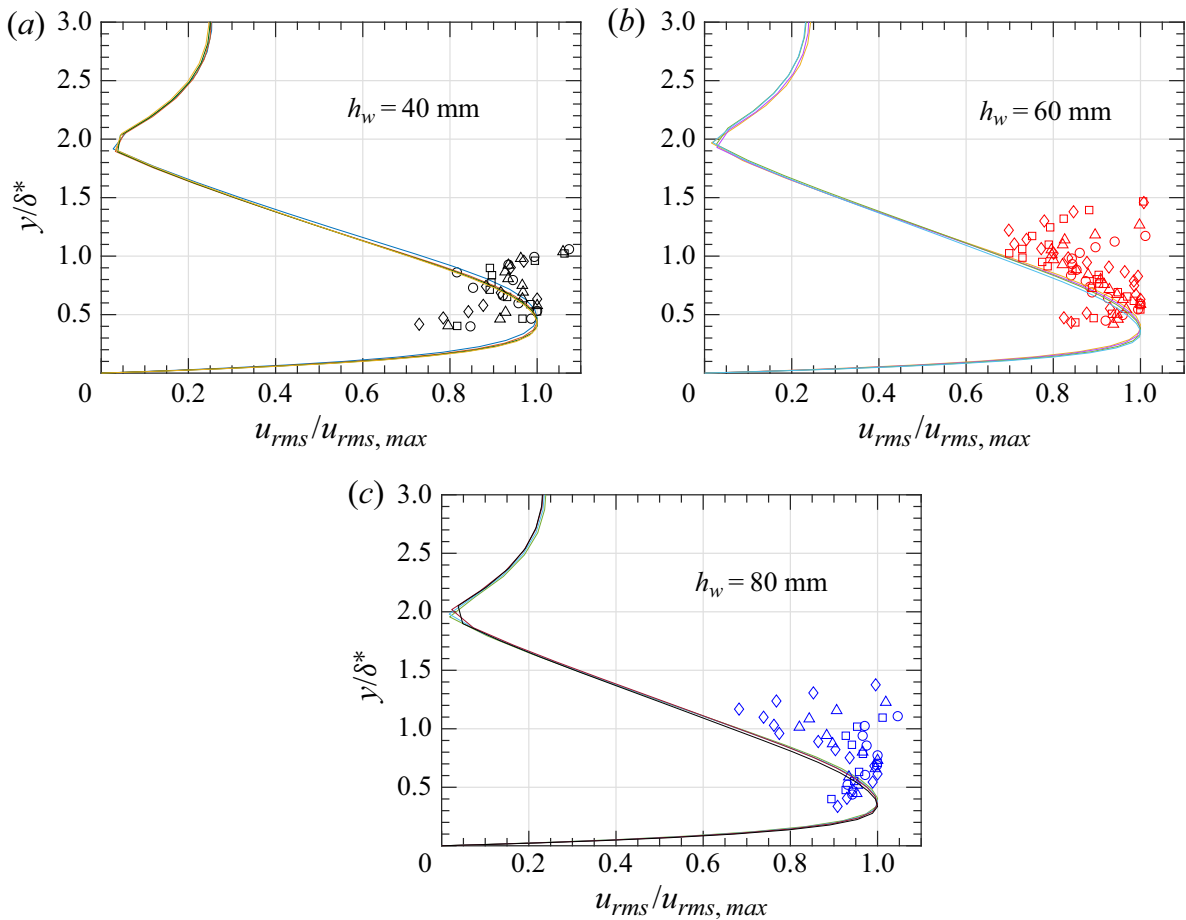

Figure 17. The $u_{r m s}$ distributions (eigenfunctions) obtained from stability calculations are compared with the filtered $u_{r m s}$ profile corresponding to the mid-range frequencies (figure 14). Solid lines obtained from the stability calculations, markers from the experiments. The legends for the markers and the solid lines are similar to those in figures 14 and $12(a-c)$ respectively.

wall-normal location $\left(y / \delta^{*}\right)$. Several authors (Kendall 1985; Westin et al. 1994; Matsubara $\&$ Alfredsson 2001) have reported the indication of streamwise streaks by strong negative correlations at a particular spanwise spacing. Such negative correlations are indicated on figure 18(a) by the dashed lines, which extend some distance downstream. By considering the time delay ( $\Delta t-$ using Taylor's hypothesis, (3.3)) between the flow travelling from one probe to the other, then the negative correlation can be shown (figure 18b) to stretch throughout the pre-transitional region. This confirms the existence of unsteady longitudinal streaky structures in the present experiment.

The wall-normal profile of the streaky structure was obtained by measuring the correlation function in the $y-z$ plane, shown in figure 19. This measurement was conducted for the case $h_{w}=40 \mathrm{~mm}$ at $x / l=0.18$, with spanwise and wall-normal step sizes of 2 $\mathrm{mm}$ and $0.1 \mathrm{~mm}$, respectively. The local boundary layer thickness was found to be $3 \mathrm{~mm}$, and from figure 19, it is evident that the streak extends up to $65 \%$ of the boundary layer thickness.

For FST-induced bypass transition, Kendall (1985) used flow visualisation to show that the spanwise distance between the origin and the location of minimum $R$ was equal to the spanwise scale of the streaky structure, or half the wavelength of the streak spacing (here, the wavelength is defined as the spanwise distance between the two consecutive positive/negative streaks). Using this definition, figure 18 shows that the spanwise scale of the streak is $30 \mathrm{~mm}$ and remains almost constant in the downstream distance, as observed in the FST case by Matsubara \& Alfredsson (2001). Further, Matsubara and Alfredsson showed that the spanwise scale of the streaks approaches the boundary layer thickness 

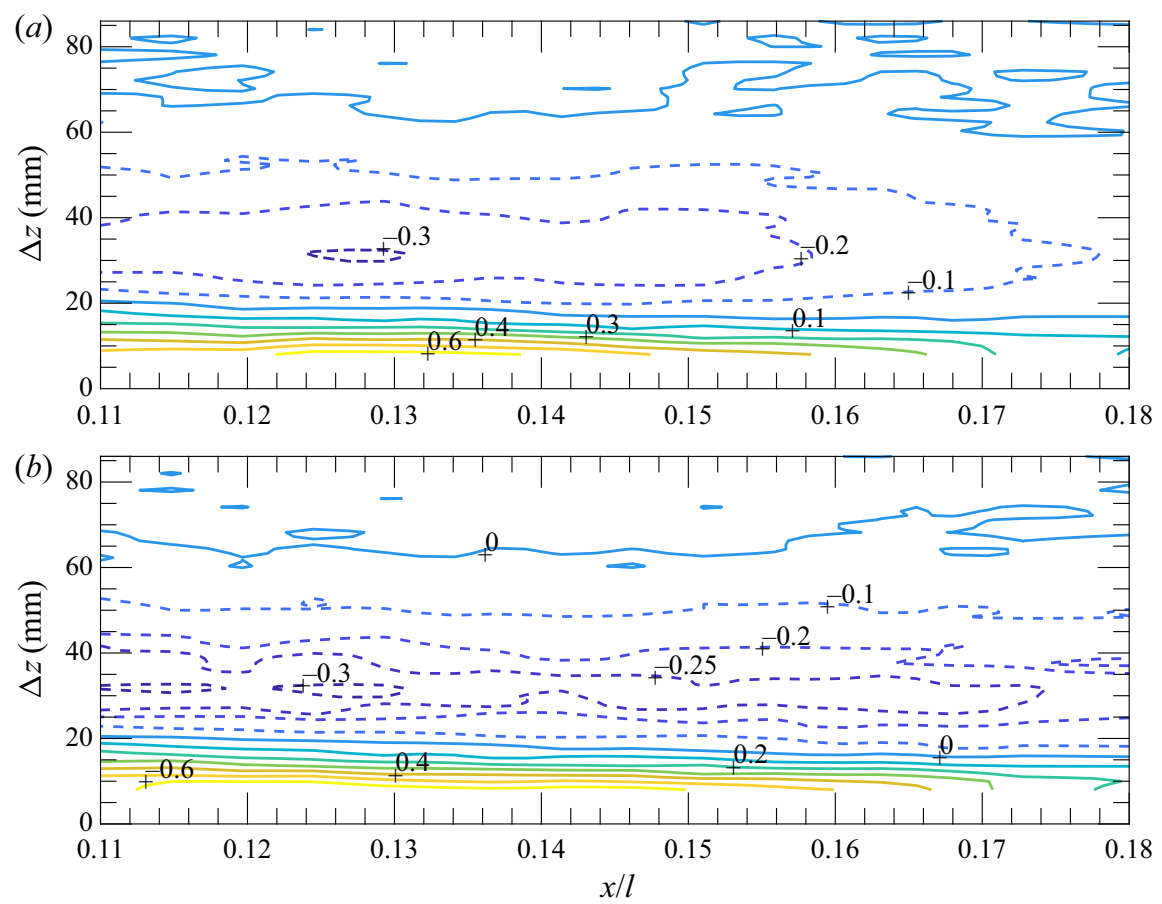

Figure 18. Contours of the streamwise correlation function $(a) R$ and $(b) R_{t}$, for the $h_{w}=40 \mathrm{~mm}$ case. Probe 1 was fixed at $x / l=0.13, y / \delta^{*}=1.3, z=0$, and probe 2 was moved in both streamwise and spanwise directions. Dashed lines represent negative correlation.

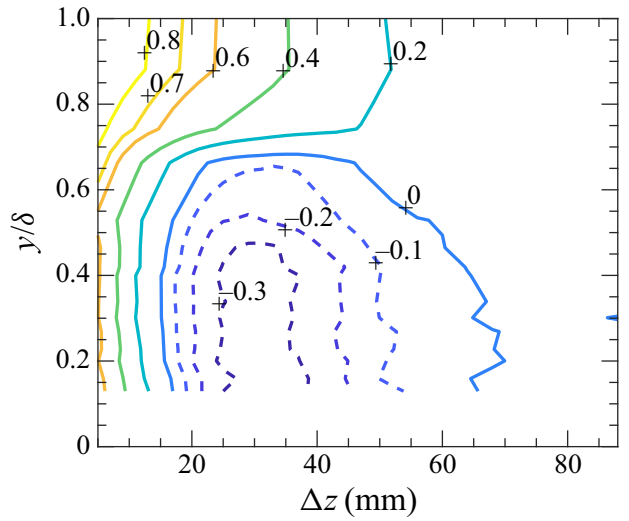

Figure 19. Wall-normal contours of the spanwise correlation for $h_{w}=40 \mathrm{~mm}, x / l=0.18$, dashed lines indicate negative correlation.

at the downstream end of the pre-transitional zone. In the case of cylinder wake-induced transition, Mandal \& Dey (2011) reported that the streak spacing was of the same order of magnitude as observed in FST-induced transition. However, in the present experiment, the spanwise scale of the streak does not approach the boundary layer thickness in the pre-transitional region: for the $h_{w}=40 \mathrm{~mm}$ case presented in figure 20(a), the minimum spanwise correlation occurs at a separation of $15 \delta$ at $x / l=0.09$, and gradually reduces to $8 \delta$ just before transition onset $(x / l=0.24)$. Hence, the streak spacing in the present 
Transition induced by the upstream aerofoil wake
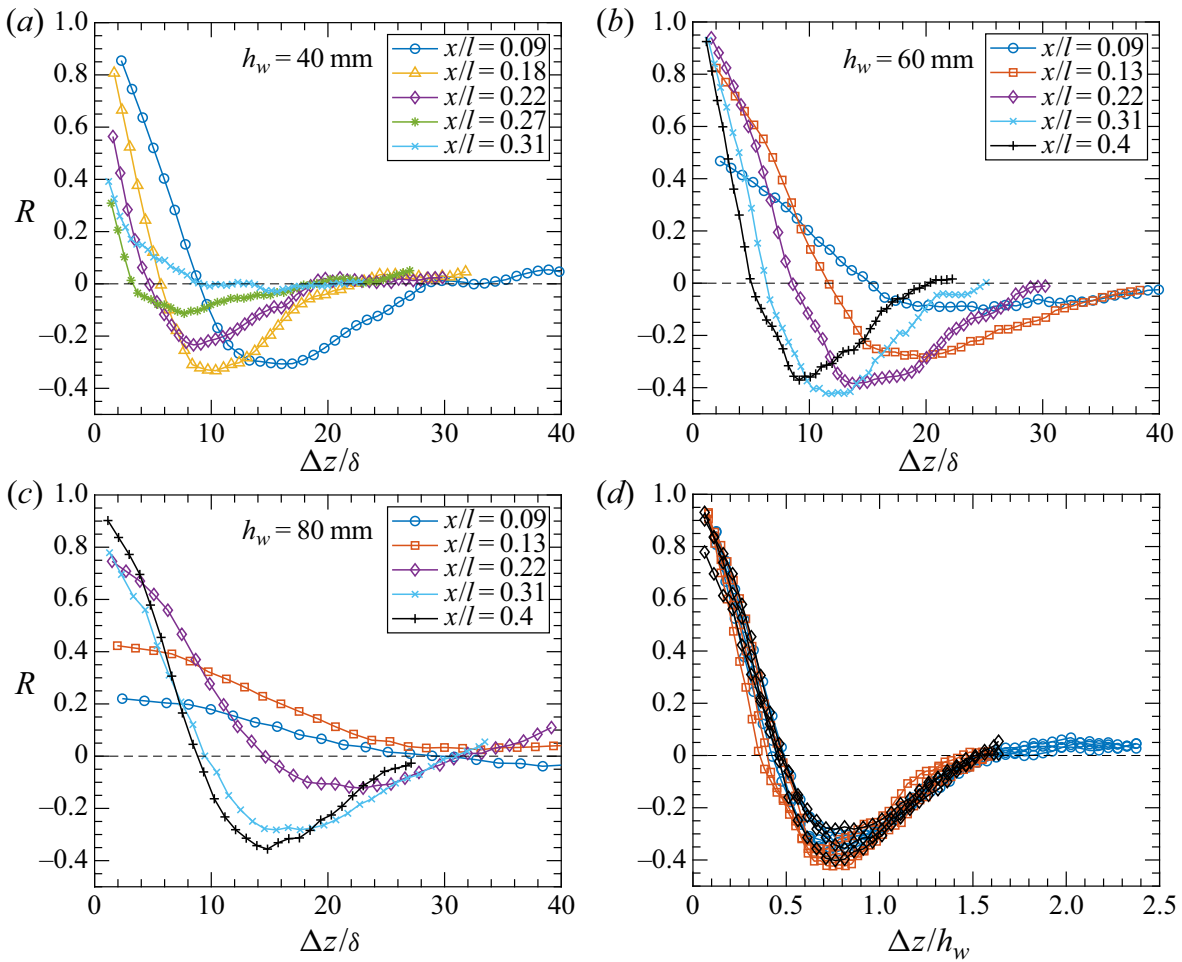

Figure 20. $(a-c)$ Spanwise correlation function $(R)$ measured at the wall-normal position close to $u_{r m s, \max }$. For these measurements $\Delta x$ was zero. $(d)$ Results from figure $20(a-c)$ are re-plotted with spanwise distance normalised with aerofoil height. Symbols: circle $-x / l=0.09,0.13$ and 0.18 for $h_{w}=40 \mathrm{~mm}$, square $-x / l=$ $0.18,0.31$ and 0.4 for $h_{w}=60 \mathrm{~mm}$, diamond $-x / l=0.31,0.35$ and 0.4 for $h_{w}=80 \mathrm{~mm}$.

experiment is much larger than the reported values in FST and cylinder wake-induced transition.

This is further confirmed by comparing the non-dimensional spanwise wavenumber ( $\beta=\beta^{*} \nu \sqrt{R e_{x}} / U_{0}$ ) with the spanwise scale of the optimal disturbance used in the theory (Andersson et al. 1999). Here, $\beta^{*}$ is referred to as dimensional spanwise wavenumber, $2 \pi /\left(2 \times \Delta z @ R_{\min }\right)$. We found that $\beta$ varies between 0.04 and 0.06 for $h_{w}=40 \mathrm{~mm}$ and it starts from 0.035 for $h_{w}=60$ and $80 \mathrm{~mm}$ at the streak located stations. This observation shows the measured $\beta$ for the current configuration is an order of magnitude lower than the optimal spanwise wavenumber proposed for FST induced transition (0.45). In addition, if the spanwise separation, $\Delta z$, is normalised with the corresponding aerofoil height, $h_{w}$, then many of the correlation functions collapse to a single curve, as shown in figure $20(d)$ (the exceptions are discussed later). This correlation between the spanwise scale of the streaky structure in the present experiment and the aerofoil height is unmistakable but unexplained, given the lack of any physical mechanism which might connect the two length scales.

We now consider the curves in figure $20(a-c)$ which do not collapse to a uniform characteristic shown in figure $20(d)$. These include the spanwise correlations at $x / l=0.09$ for $h_{w}=60 \mathrm{~mm}$ (most upstream curve in figure 20b) and at $x / l=0.09$ and 0.13 for $h_{w}=80 \mathrm{~mm}$ (most upstream two curves in figure 20c). The curve for $x / l=0.22$ for $h_{w}=80 \mathrm{~mm}$ also displays a smaller peak negative correlation when compared with the downstream stations. These outliers are all consistent with the variation in $u_{r m s}$ profiles 


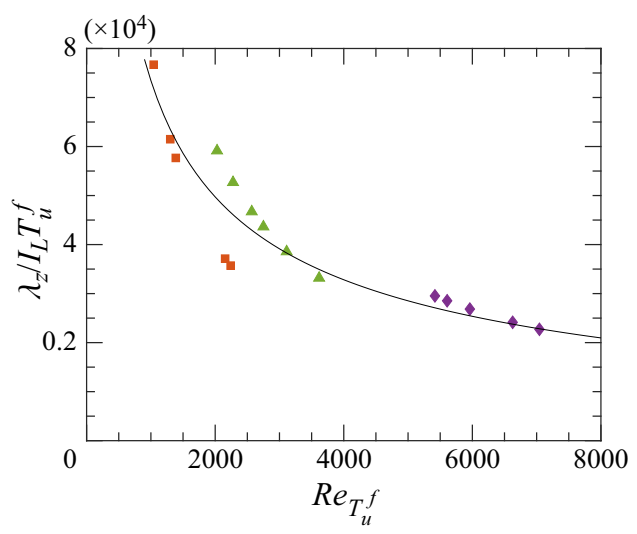

Figure 21. Spanwise wavelength of the streak normalised with the product of forcing turbulence and integral length scale at the boundary layer edge.

shown in figure 6, where the disturbance profiles at $x / l=0.09$ for $h_{w}=60 \mathrm{~mm}$ and $x / l<0.31$ for $h_{w}=80 \mathrm{~mm}$ deviate from the majority, non-modal distributions. It is clear from figure 20 that the origin of the streaky structure gradually moves downstream as the aerofoil is moved further from the flat plate: for the $h_{w}=80 \mathrm{~mm}$ case the genesis of the streaky structure is quite gradual. In the case of FST-induced bypass transition, the longitudinal streaky structure originates from the leading edge (Kendall 1985; Westin et al. 1994), and in the case of a cylinder wake, the streaky structure follows immediately after the destabilisation of the secondary spanwise vortex in the boundary layer (Pan et al. 2008; Mandal \& Dey 2011). However, in the present experiment, it is clear that the proximity of the wake controls the origin of the streaky structure in a progressive manner.

As mentioned previously, the reason for the collapse shown in figure $20(d)$ is unknown, but varying the aerofoil height alters the two characteristics of the forcing, forcing turbulence $\left(T_{u}^{f}, I_{L}\right)$ and forcing frequency from the wake. In a recent article by Fransson \& Shahinfar (2020) on FST-induced transition demonstrates that the spanwise scale depends on the free-stream turbulence characteristics and not on the boundary layer thickness. Moreover, the present study (figure $20 a-c$ ) also demonstrates that the spanwise scale is much larger than the boundary layer thickness and does not adapt with $\delta$. We now proceed along the lines of Fransson \& Shahinfar (2020) to see if any more instructive correlations can be revealed. For this analysis, the forcing turbulence and the integral length scale obtained at the edge of the boundary layer edge are considered as primary variables, as opposed to the free-stream turbulence considered in the article by Fransson \& Shahinfar (2020). By plotting the Reynolds number of the forcing disturbance $\left(R e_{T_{u}^{f}}=\right.$ $\left.T_{u}^{f} \times\left[U_{0} I_{L} / \nu\right]\right)$ against the spanwise wavelength $\left(\lambda_{z}=2 \times \Delta Z @ R_{\min }\right)$ normalised with $T_{u}^{f}$ and $I_{L}$, a clear trend is revealed in figure 21 . The fitted curve for the data points is given by (3.4), but its coefficients are different from Fransson and Shahinfar, probably associated with the difference in forcing characteristics. However, it confirms that the spanwise scale in the aerofoil wake-induced transition is determined by the forcing turbulence penetrating through the edge of the boundary layer

$$
\lambda_{z} / I_{L} T_{u}^{f}=\left(1477 R e_{T_{u}^{f}}^{-0.2}-100\right)^{2} .
$$




\section{Concluding remarks}

This paper attempts to characterise the early stages of laminar-turbulent transition induced by the interaction of an upstream, non-impinging turbulent wake with a downstream flat-plate boundary layer. This is believed to be the first kind of transition study using an aerofoil-flat-plate configuration. Experiments were conducted at a fixed wake Reynolds number, $R e_{c}=3.4 \times 10^{5}$ (based on aerofoil chord) for four different aerofoil heights, $h_{w}=20,40,60$ and $80 \mathrm{~mm}$, the most interesting features being observed at the three larger separations. The aerofoil was tripped to avoid large-scale coherent structures appearing in the fully turbulent wake. The turbulence intensity experienced by the laminar boundary layer was found to vary with aerofoil height: for $h_{w}=40 \mathrm{~mm}$, the interaction occurred at moderate to high intensity $(0.35 \%-1.2 \%)$, for $h_{w}=60 \mathrm{~mm}$ at moderate intensity $(0.1 \%-0.4 \%)$ and for $h_{w}=80 \mathrm{~mm}$ at low $(<0.1 \%)$ to moderate intensity. The forcing was broadband but dominated by a mid-range of frequencies $(20 \leq F \leq 100)$ which varied with aerofoil height, but did not change with streamwise distance. The key observations from the measurements obtained in the pre-transitional zone $(\gamma=0)$ are summarised below.

(i) For the lower aerofoil height $\left(h_{w}=40 \mathrm{~mm}\right)$, the $u_{r m s}$ distributions in the pre-transitional region followed the non-modal disturbance profile obtained from the transient growth theory. Increasing the aerofoil height $\left(h_{w}=60\right.$ and $\left.80 \mathrm{~mm}\right)$ resulted in an initially modal disturbance profile, which then evolved into a non-modal profile (figure 6).

(ii) The change from modal to non-modal behaviour was found to occur at approximately $T_{u}^{f}=0.1 \%$ (figure $7 a$ ).

(iii) Inside the pre-transitional boundary layer the maximum disturbance energy $\left(u_{r \operatorname{ms} \text { max }}^{2}\right)$ increased exponentially with streamwise distance (figure 8) even when the disturbance profile displayed non-modal characteristics. This contrasts with the results of transient growth theory for which non-modal disturbance energy growth is algebraic, also substantiated in the literature for FST and cylinder wake-induced transition.

(iv) Both spectral (figures 11 and 12) and $u_{r m s}$ distributions (figure 13) demonstrated the coexistence of both modal and non-modal disturbances.

(v) Two-point correlation measurements confirmed the presence of longitudinal streaky structures in the pre-transitional zone. The average spanwise scale of the streak was found to be larger than the boundary layer thickness, $(>8 \delta)$, figure 20 .

(vi) Increasing the aerofoil height shifts the origin of the streaky structure downstream, figure 20 .

(vii) In the region of non-modal disturbances, the two-point spanwise correlation functions obtained for different aerofoil heights $\left(h_{w}\right)$ were found to collapse when the spanwise distance was normalised with $h_{w}$, figure $20(d)$, indicating that the spanwise scale of the longitudinal streaks was proportional to $h_{w}$.

(viii) The spanwise scale of the streaky structure is found to correlate with the forcing turbulence characteristics $\left(T_{u}^{f}, I_{L}\right)$ penetrating through the boundary layer edge.

Having compared the present observations with the transition characteristics of natural, bypass and cylinder wake-boundary layer interaction, it is concluded that the features observed in the current experiments do not correspond to one particular type of transition. Rather, the present results show a mixed transitional behaviour, attributed to the particular characteristics of the aerofoil-wake boundary layer interaction. The trends support the 


\section{Veerasamy, C.J. Atkin, and S.A. Ponnusami}

idea that, when the aerofoil height is increased, the transition characteristics shift from non-modal to modal, with mixed characteristics for intermediate aerofoil heights. Further, the present experiment revealed differences in the energy growth trends (exponential vs algebraic) plus greater, and forcing-related, spacing of the streaky structure compared with those reported in existing studies on non-modal disturbances, such as cylinder wake and FST-induced transition.

The experimental arrangement offers an attractive method of controlling the forcing environment so as to investigate the receptivity mechanism and the evolution of non-modal disturbance profiles in greater detail. In addition to refinement and verification of the results presented here, further work could include exploring the effect of lift on the upstream aerofoil as well as pressure gradient on the downstream boundary layer.

Acknowledgements. We thank Professor M. Gaster for his comments, suggestion and fruitful discussion during this work. Also the first author is grateful to Dr A. Mitra and Mr J. Varghese for their help in stability calculation.

Funding. This work was supported by Innovate UK under Grant Ref. 113024, Enhanced Fidelity Transonic Wing, led by Airbus.

Declaration of interest. The authors report no conflict of interest.

Author ORCIDs.

(D) Dhamotharan Veerasamy https://orcid.org/0000-0001-9511-6281;

(D) Chris J. Atkin https://orcid.org/0000-0003-2529-1978.

\section{Appendix A}

Linear stability analysis is carried out by solving the classical Orr-Sommerfeld equation shown in (A1). Here, $\hat{v}$ is the amplitude of the wave-like perturbation as a function of wall-normal distance, $\hat{v}=\hat{v}(y) \mathrm{e}^{\mathrm{i} \alpha x-\omega t}$. Further, $\alpha, \omega, c$ and $\operatorname{Re}_{\delta^{*}}$ represent the wavenumber in the streamwise direction, angular frequency, phase velocity $(\omega / \alpha)$ and Reynolds number based on displacement thickness, respectively. By rearranging (A1), the Orr-Sommerfeld equation can be cast as an eigenvalue problem (A2), particularly for the present study (wake-boundary layer) considered as a spatial eigenvalue problem. Upon imposing the boundary conditions $\left(\hat{v}=\hat{v}^{\prime}=0\right.$ at the solid wall and far field) it is solved for complex wavenumber $\left(\alpha=\alpha_{r}+\mathrm{i} \alpha_{i}\right)$, by considering $\omega$ to be real

$$
\begin{gathered}
{\left[(\mathrm{i} U \alpha-\mathrm{i} \omega)\left(D^{2}-\alpha^{2}\right)-\mathrm{i} \alpha U^{\prime \prime}-\frac{1}{\operatorname{Re}_{\delta^{*}}}\left(D^{2}-\alpha^{2}\right)^{2}\right] \hat{v}=0,} \\
{\left[\mathrm{i} U\left(D^{2}-\alpha^{2}\right)-\mathrm{i} U^{\prime \prime}-\frac{1}{\alpha \operatorname{Re}_{\delta^{*}}}\left(D^{2}-\alpha^{2}\right)^{2}\right] \hat{v}=\mathrm{i} c\left(D^{2}-\alpha^{2}\right) \hat{v} .}
\end{gathered}
$$

The method used for the present analysis has been validated with a rather relevant analysis conducted by Liou \& Liu (2001) on the confluent wake-boundary layer. Following their approach, a base flow velocity profile is modelled by superposition of the Blasius boundary layer profile and the Gaussian wake profile $\left(U=1-0.6 e^{-0.5\left(y-h_{w}\right)^{2}}\right)$, shown in figure 22(a). By considering the same geometry of the wake, $R_{\delta^{*}}$ and $\omega$ as reported in Liou \& Liu (2001), the Orr-Sommerfeld equation is solved using the approach indicated earlier and three discrete unstable modes are obtained: one boundary layer mode and two wake modes (shown in figure 22b). The wake modes 1 and 2 correspond to antisymmetric and symmetric modes, respectively. These three unstable modes are also reported by Liou \& Liu (2001). To validate further, the eigenfunctions corresponding to the three unstable modes are compared and are shown in figure 23. 
Transition induced by the upstream aerofoil wake
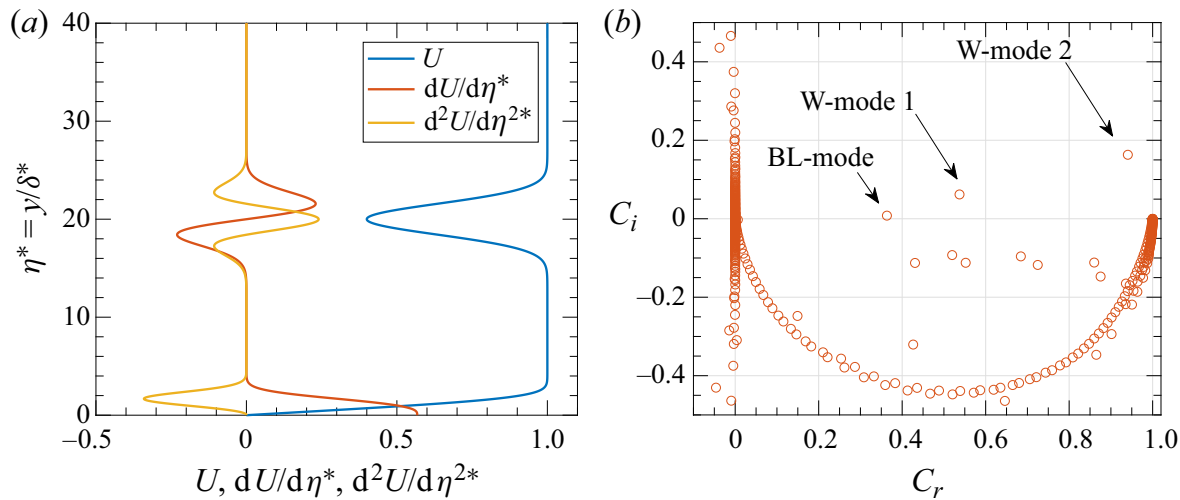

Figure 22. (a) Wake-boundary layer profile and its derivative, as similar in Liou \& Liu (2001). (b) Corresponding eigenvalue spectrum obtained at $R e_{\delta^{*}}=998$ and $\omega=0.112$. BL- and W-mode stands for boundary layer and wake modes.
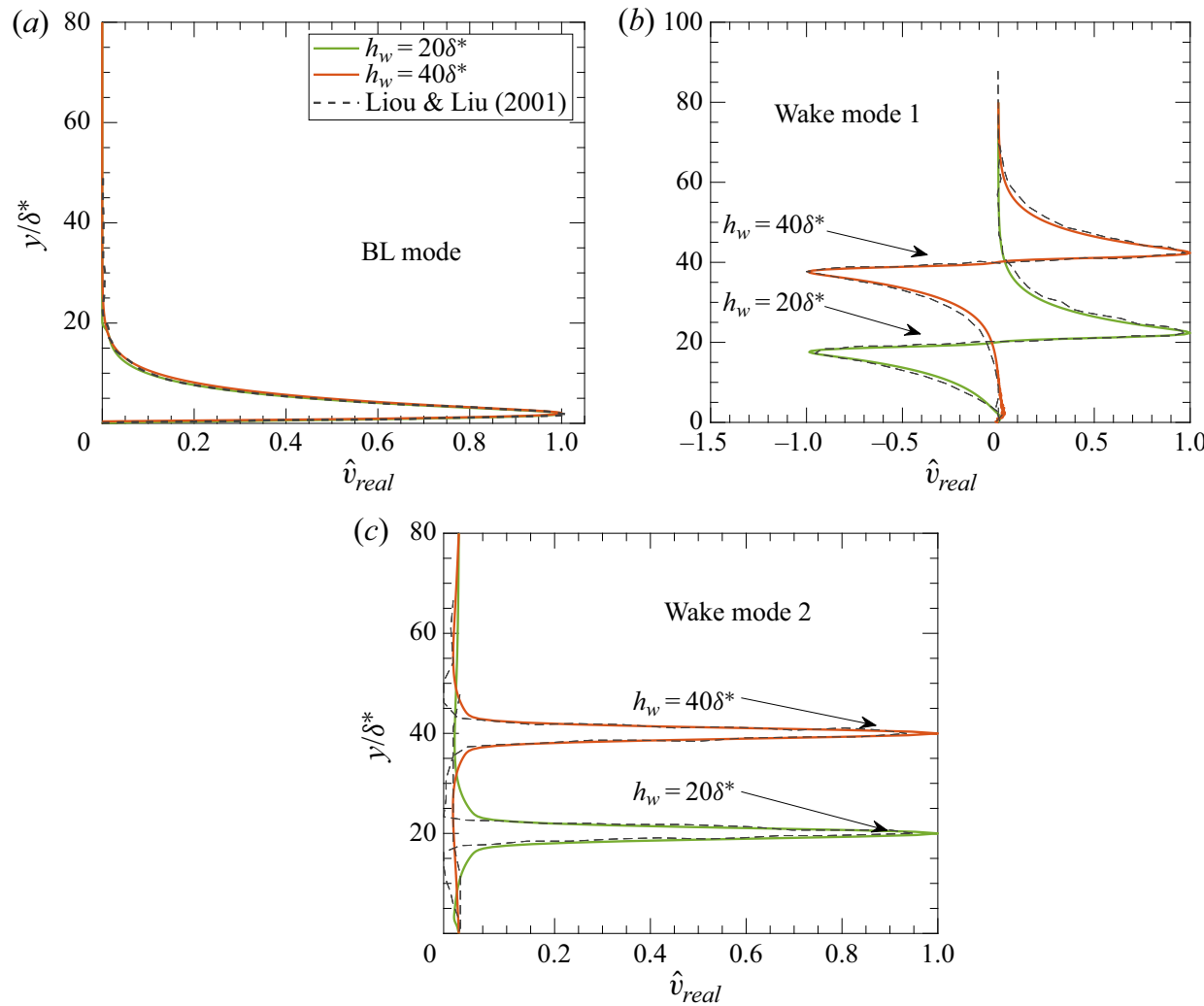

Figure 23. Eigenfunctions of ( $a$ ) boundary layer mode $(b)$ wake mode- 1 and $(c)$ wake mode- 2 for the two different wake heights, $h_{w} / \delta^{*}=20$ and $40 \mathrm{~mm}$ at $R e_{\delta^{*}}=998, \omega=0.112$. 
(a)

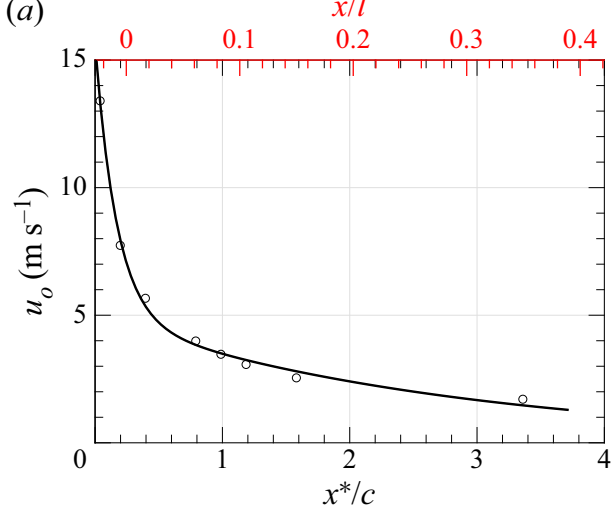

(b)

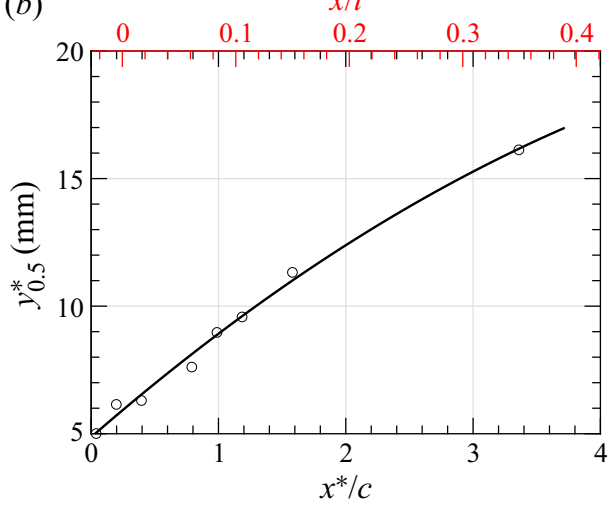

Figure 24. (a) Decay of defect velocity, and $(b)$ position of $0.5 u_{0}$ of the wake in the downstream distance.

\section{Appendix B}

To obtain a smooth velocity profile for the stability analysis (i.e. without any discontinuities in the derivatives), a confluent wake-boundary layer profile is generated numerically to match the measured wake profile. There is a considerable potential core between the wake region and boundary layer for the case $h_{w}=60 \mathrm{~mm}$. The potential core suggests that the wake and the boundary layer have minimal effect on each other in a time-averaged sense. From this observation, it is assumed that the downstream wake growth is not distorted due to the boundary layer. Therefore, in order to obtain the combined wake-boundary layer profile, the velocity profiles of the aerofoil wake and the boundary layer are superposed. The procedure followed for the superposition is described below

$$
\begin{gathered}
y_{0.5}=-7.7 \times 10^{-6}(x / l)^{2}+0.024(x / l)+4.1, \\
u_{0}=6.26 \exp [-0.00648(x / l)]+2.506 \exp [-0.000468(x / l)], \\
f(\eta)=\frac{U_{0}-\bar{U}}{u_{0}}=\exp \left[-0.637(\eta)^{2}-0.056(\eta)^{4}\right] .
\end{gathered}
$$

From the data measured for the pure aerofoil wake, reported in $\S 2$, the characteristics of the aerofoil wake are obtained in terms of development of defect velocity and wake thickness in the downstream direction, which are shown in figure 24. Through a curve fitting technique, expressions for wake thickness and defect velocity can be obtained as given in (B1) and (B2), respectively. With this information, and using the self-similarity equation for aerofoil wake given by (B3) (Wygnanski et al. 1986), velocity profiles of the wake at any streamwise station can be obtained. From $\S 2$, it is known that the boundary layer profiles for case $h_{w}=60 \mathrm{~mm}$ match the Blasius solution. Having the velocity profiles for the wake and the boundary layer, the velocity profile of the combined wake-boundary layer can be obtained.

\section{REFERENCES}

Andersson, P., Berggren, M. \& Henningson, D.S. 1999 Optimal disturbances and bypass transition in boundary layers. Phys. Fluids 11 (1), 134-150.

Balamurugan, G. \& MAndal, A.C. 2017 Experiments on localized secondary instability in bypass boundary layer transition. J. Fluid Mech. 817, 217-263. 


\section{Transition induced by the upstream aerofoil wake}

BLAIR, M.F. 1992 Boundary-layer transition in accelerating flows with intense freestream turbulence: part 2- the zone of intermittent turbulence. Trans. ASME J. Fluids Engng 114 (3), 322-332.

Coull, J.D. \& Hodson, H.P. 2011 Unsteady boundary-layer transition in low-pressure turbines. J. Fluid Mech. 681, 370-410.

Delattre, G., BRAZier, J.P., Forte, M. \& PASCAL, L. 2018 Impact of upstream wake on boundary layer regime: from modal to bypass transition. In Fluid Dynamics Conference, AIAA Paper 2018-3387.

Dixit, S.A. \& RAMESH, O.N. 2010 Large-scale structures in turbulent and reverse-transitional sink flow boundary layers. J. Fluid Mech. 649, 233-273.

Durbin, P. \& Wu, X.H. 2007 Transition beneath vortical disturbances. Annu. Rev. Fluid Mech. 39, 107-128.

FASEL, H.F. 2002 Numerical investigation of the interaction of the Klebanoff-mode with a Tollmien-Schlichting wave. J. Fluid Mech. 450, 1-33.

Fransson, J.H.M., Matsubara, M. \& Alfredsson, P.H. 2005 Transition induced by free-stream turbulence. J. Fluid Mech. 527, 1-25.

FRANSSON, J.H.M. \& SHAHINFAR, S. 2020 On the effect of free-stream turbulence on boundary-layer transition. J. Fluid Mech. 899, A23.

HAJ-HARIRI, H. 1988 Transformations reducing the order of the parameter in differential eigenvalue problems. J. Comput. Phys. 77 (2), 472-484.

HE, G., WANG, J. \& PAN, C. 2013 Initial growth of a disturbance in a boundary layer influenced by a circular cylinder wake. J. Fluid Mech. 718, 116-130.

HE, G.-S., PAN, C., FENG, L.-H., GAO, Q. \& WANG, J.-J. 2016 Evolution of Lagrangian coherent structures in a cylinder-wake disturbed flat plate boundary layer. J. Fluid Mech. 792, 274-306.

Hodson, H.P. \& HoweLL, R.J. 2005 Bladerow interactions, transition, and high-lift aerofoils in low-pressure turbines. Аnпи. Rev. Fluid Mech. 37, 71-98.

Hourmouziadis, J. 1989 Aerodynamic design of low pressure turbines. AGARD, Lecture Series No. 167.

JACOBS, R.G. \& DURBIN, P.A. 1998 Shear sheltering and the continuous spectrum of the Orr-Sommerfeld equation. Phys. Fluids 10 (8), 2006-2011.

Kenchi, T., Matsubara, M. \& IKeda, T. 2008 Laminar turbulent transition in a boundary layer subjected to weak free stream turbulence. J. Fluid Sci. Technol. 3 (1), 56-67.

KendALL, J. 1985 Experimental study of disturbances produced in a pre-transitional laminar boundary layer by weak freestream turbulence. In 18th Fluid Dynamics and Plasma Dynamics and Lasers Conference, AIAA Paper 1985-1695.

Kosorygin, V.S. \& Polyakov, N.P. 1990 Laminar boundary layers in turbulent flows. In IUTAM, Laminar-Turbulent Transition (ed. D. Arnal \& R. Michel), pp. 573-578. Springer.

KUAN, C.L. \& WANG, T. 1990 Investigation of the intermittent behavior of transitional boundary layer using a conditional averaging technique. Exp. Therm. Fluid Sci. 3 (2), 157-173.

KYRIAKIDES, N.K., KAStRinaKis, E.G., NYChas, S.G. \& Goulas, A. 1999 Aspects of flow structure during a cylinder wake-induced laminar/turbulent transition. AIAA J. 37 (10), 1197-1205.

LI, Y. \& GASTER, M. 2006 Active control of boundary-layer instabilities. J. Fluid Mech. 550, 185-205.

LIOU, W.W. \& LIU, F. 2001 Spatial linear instability of confluent wake/boundary layers. AIAA J. 39 (11), 2076-2081.

LUCHINI, P. 2000 Reynolds-number-independent instability of the boundary layer over a flat surface: optimal perturbations. J. Fluid Mech. 404, 289-309.

MANDAL, A.C. \& DEY, J. 2011 An experimental study of boundary layer transition induced by a cylinder wake. J. Fluid Mech. 684, 60-84.

MATSUbARA, M. \& ALFREDSSON, P.H. 2001 Disturbance growth in boundary layers subjected to free-stream turbulence. J. Fluid Mech. 430, 149-168.

MitrA, A. 2018 Dynamics of laminar separation bubbles on low Reynolds number airfoils. PhD thesis, Indian Institute of Science.

Norimatsu, R., TAKai, S. \& Matsubara, M. 2011 Relation between disturbance of boundary layer and free stream turbulence component. In ASME-JSME-KSME 2011 Joint Fluids Engineering Conference, pp. 2987-2992. American Society of Mechanical Engineers.

Ovchinnikov, V., Piomelli, U. \& Choudhari, M.M. 2006 Numerical simulations of boundary-layer transition induced by a cylinder wake. J. Fluid Mech. 547, 413-441.

PAN, C., WANG, J.J., Zhang, P.F. \& FEnG, L.H. 2008 Coherent structures in bypass transition induced by a cylinder wake. J. Fluid Mech. 603, 367-389.

Pfeil, H. \& HeRbST, R. 1979 Transition procedure of instationary boundary layers. In ASME 1979 International Gas Turbine Conference and Exhibit and Solar Energy Conference. Volume 1B: Gas Turbines. American Society of Mechanical Engineers Digital Collection.

Pope, S.B. 2000 Turbulent Flows. Cambridge University Press. 


\section{Veerasamy, C.J. Atkin, and S.A. Ponnusami}

SChmid, P.J. \& Henningson, D.S. 2012 Stability and Transition in Shear Flows, vol. 142. Springer Science \& Business Media.

Smith, A.M.O. \& Gamberoni, A.H. 1956 Transition, pressure gradient and stability theory. Douglas Aircraft Co., Report ES 26388.

SPAID, F.W. 2000 High Reynolds number, multielement airfoil flowfield measurements. J. Aircraft 37 (3), 499-507.

SQUIRE, L.C. 1989 Interactions between wakes and boundary-layers. Prog. Aerosp. Sci. 26 (3), 261-288.

VAN INGEN, J.L. 1956 A suggested semi-empirical method for the calculation of the boundary layer transition region. Technische Hogeschool Delft, Vliegtuigbouwkunde, Rapport VTH-74.

VARGHESE, J. 2016 Linear instability and transition of incompressible plane wall jet. Master's thesis, Indian Institute of Science.

VEERASAMY, D. 2019 Effect on flap transition of upstream wake turbulence. PhD thesis, City, University of London.

Veerasamy, D. \& AtKin, C. 2020 A rational method for determining intermittency in the transitional boundary layer. Exp. Fluids 61 (1), 11.

Westin, K.J.A., Boiko, A.V., Klingmann, B.G.B., Kozlov, V.V. \& Alfredsson, P.H. 1994 Experiments in a boundary layer subjected to free stream turbulence. Part 1. Boundary layer structure and receptivity. J. Fluid Mech. 281, 193-218.

Wygnanski, I., Champagne, F. \& Marasli, B. 1986 On the large-scale structures in two-dimensional, small-deficit, turbulent wakes. J. Fluid Mech. 168, 31-71. 